\title{
A Mathematical Model Analysis of Age Structured Malaria Transmission
}

\author{
Selashi Dabi Lole Tadese Lamesa Edae \\ College of Natural and Computational science, \\ Department of Applied Mathematics, Woalita sodo university, sodo Ethiopia,P.O.BOX .138
}

\begin{abstract}
Mathematical models have been used to provide an explicit framework for understanding malaria transmission dynamics in human population for over 100 years. The equilibrium points of the model are found and their stability is investigated. By analyzing the model, a threshold parameter $R_{0}$ was found which the basic reproductive number is obtained. It is noted that when $R_{0}<1$ the disease will fail to spread and when $R_{0}>1$ the disease will persist in the population and become endemic. The stability of these two equilibrium points is controlled by the basic reproductive number $R_{0}$ which was determined using the next generation matrix approach. Our model divides the human population into three compartments: pre-school (0-5 years) and youngest age group ( $6-25$ years old) and the rest of the human population. The results of our mathematical analysis indicate that the disease-free equilibrium is asymptotically stable and occurs whenever the basic reproduction number $R_{0}$ is less than unity or
\end{abstract} $R_{0}<1$. For $R_{0}>1$, the disease-free equilibrium point is unstable.

Keywords: Mathematical model, equilibrium point, Basic Reproductive number, Stability DOI: $10.7176 / \mathrm{MTM} / 9-5-04$

Publication date:May $31^{\text {st }} 2019$

\section{INTRODUCTION}

In order to reflect the age structure of a population affects the dynamics of disease transmission, we incorporate age into the model, and assume that the infection rate depend on the age. We model observations to be able to describe naturally occurring phenomena, to be able to predict future events and to be able to find optimal solutions to problems we encounter.

That first malaria model was expanded in several ways, for example by introducing the effects of human age [21], acquired immunity to malaria [17,21] or genetic and special heterogeneity of parasite and host among these age and immunity play an important role in endemic malaria regions. In order to describe age-structured models for disease transmission we must first develop the theory of age-structured populations. In fact, the first models for age-structured populations were designed for the study of disease transmission in such populations. In this paper, we construct a new model to investigate the patterns of malaria transmission in the presence of age structure model.

\section{Disease Free Equilibrium point}

Disease-free equilibrium points $(D F E)$ are steady state solutions where there is no malaria in the human population or Plasmodium parasite in the mosquito population. In absence of the disease, this implies that $i_{h 1}, i_{h 2}, i_{h 3}, i_{v}=0$ To determine the stability of the equilibrium points of the given model we evaluate the steady state of the given system with following conditions.

$>$ Disease free equilibrium points $i_{h 1}, i_{h 2}, i_{h 3}, i_{v}=0$

\subsection{Endemic disease equilibrium point}

The equilibrium point at the endemic level has a strong influence in the behavior of the disease transmission for a given community. The endemic equilibrium point is the final reachable situation, even though there could be infinitely many different initial distributions of malaria in a community. Stability analysis is an important mathematical tool that provides the conditions that guarantee the stability of an endemic equilibrium point. In the presence of the disease, this implies that $i_{h 1}, i_{h 2}, i_{h 3}, i_{v} \neq 0$

To determine the stability of the equilibrium points of the given model we evaluate the steady state of the given system with following conditions.

$>$ Endemic equilibrium points, that is $i_{h 1}, i_{h 2}, i_{h 3}, i_{v} \neq 0$ 


\subsection{Basic concept of reproductive number $R_{0}$}

A quantity of central importance in epidemiology is the basic reproduction number denoted by $R_{0}$. From time to time, people also call it the basic reproductive rate or ratio, or the basic reproduction ratio. $R_{0}$ is defined as the mean number of secondary infections produced when one infected individual is introduced into a host population where everyone is susceptible.

As defined, a reproductive number less than one will cause the disease to die out; whereas, a reproductive number greater than one classifies the disease as endemic [2]i.eif $R_{0}<1$, then on average an infected individual produces less than one new infected individual over the course of its infectious period, and the infection cannot grow. Conversely, if $R_{0}>1$, then each infected individual produces, on average, more than one new infection, and the disease can invade the population.

\subsection{Next generation matrices for compartmental epidemic model}

The basic reproduction number $R_{0}$ is arguably the most important quantity in infectious disease epidemiology.

The next-generation matrix (NGM) is the natural basis for the definition and calculation of $R_{0}$ where finitely many different categories of individuals are recognized.

In epidemiology, the next-generation matrix is a method used to derive the basicreproduction number, for a compartmental model of the spread of infectious diseases. This method is given by Diekmannet al. (1990)[12]and Driessche and Watmough (2002).

To calculate the basic reproduction number by using a next-generation matrix, the whole population is divided into $n$ compartments in which there are $m<n$ infected compartments. Let $x_{i}, i=1,2,3,4, \ldots, m$ be the numbers of infected individuals in the $i^{\text {th }}$ infected compartment at time $t$.

\subsection{Routh-Hurwitz Criteria}

1.4.1. General Procedure

The Routh-Hurwitz (RH) Criterion is a general mathematical technique thatmay be used to determine how many of the roots of a characteristic equationsuch as the one below have positive real parts, and are therefore unstable1.Routh-Hurwitiz is a method for determining whether a linear system is stable or not by examining the location of the roots of the characteristic equation of the system.

The Routh-Hurwitz Criteria do not yield information about the absolute values of the eigenvalues, butabout their signs. For checking for stability, it is sufficient to know the sign of the real parts.

The Consider the general form for a characteristic polynomial

$$
p(\lambda)=\lambda^{n}+a_{n-1} \lambda^{n-1}+a_{n-2} \lambda^{n-2}+a_{n-3} \lambda^{n}+\ldots+a_{1} \lambda+a_{0}
$$

To determine whether the system stable or not, check the following conditions.

1. Two necessary but not sufficient conditions that all the roots have negative real parts are:

a) All the polynomial coefficients must have the same sign.

b) All the polynomial coefficients must be nonzero.

2. If this condition is satisfied then compute the Routh -Hurwitz array as follows:

$$
\begin{aligned}
& \lambda^{n} a_{n} a_{n-2} a_{n-4} a_{n-6} \cdots \\
& \lambda^{n-1} a_{l-1} a_{n-3} a_{n-5} a_{n-7} \cdots \\
& \lambda^{n-2} b_{1} b_{2} b_{3} b_{4} \cdots \\
& \lambda^{n-3} k_{1} k_{2} k_{3} k_{4} \cdots \\
& \lambda^{0 !}
\end{aligned}
$$

Where the aicoefficients come from the original polynomial. The other coefficients are computed in a fashion similar to a determinant, by selecting appropriate values in the array. The pattern of values is illustrated via the following cases:

The associated Routh Array is then: 


\begin{tabular}{|l|l|l|l|l|}
\hline$\lambda^{n}$ & $a_{n}$ & $a_{n-2}$ & $a_{n-4}$ & $\cdots$ \\
\hline$\lambda^{n-1}$ & $a_{n-1}$ & $a_{n-3}$ & $a_{n-5}$ & $\cdots$ \\
\hline$\lambda^{n-2}$ & $b_{1}$ & $b_{2}$ & $b_{3}$ & $\cdots$ \\
\hline$\lambda^{n-3}$ & $k_{1}$ & $k_{2}$ & $k_{3}$ & \\
\hline$\cdots$ & & & \\
\hline$\lambda^{0}$ & \multicolumn{4}{|c}{ Where } \\
\hline \multicolumn{4}{|c|}{} \\
\hline
\end{tabular}

$$
\begin{gathered}
b_{1}=\frac{-1}{a_{n-1}}\left|\begin{array}{cc}
a_{n} & a_{n-2} \\
a_{n-1} & a_{n-3}
\end{array}\right|=\frac{-1}{a_{n-1}}\left(a_{n} a_{n-3}-a_{n-2} a_{n-1}\right) \\
b_{2}=\frac{-1}{a_{n-1}}\left|\begin{array}{ll}
a_{n} & a_{n-4} \\
a_{n-1} & a_{n-5}
\end{array}\right|=\frac{-1}{a_{n-1}}\left(a_{n} a_{n-5}-a_{n-4} a_{n-1}\right) \\
b_{3}=\frac{-1}{a_{n-1}}\left|\begin{array}{cc}
a_{n} & a_{n-6} \\
a_{n-1} & a_{n-7}
\end{array}\right|=\frac{-1}{a_{n-1}}\left(a_{n} a_{n-7}-a_{n-6} a_{n-1}\right) \\
k_{1}=\frac{-1}{b_{1}}\left|\begin{array}{cc}
a_{n-1} & a_{n-3} \\
b_{1} & b_{2}
\end{array}\right|=\frac{-1}{b_{1}}\left(a_{n-1} b_{2}-b_{1} a_{n-3}\right) \\
k_{2}=\frac{-1}{b_{1}}\left|\begin{array}{cc}
a_{n-1} & a_{n-5} \\
b_{1} & b_{3}
\end{array}\right|=\frac{-1}{b_{1}}\left(a_{n-1} b_{3}-b_{1} a_{n-5}\right)
\end{gathered}
$$

\section{THE BASICS MODEL}

These categories or compartments are represented by standard notation of $S-E-I-R$ after the pioneering work of Kermack and McKendrick. There may be variations in the compartment structure depending on the type of disease. For example, the Iclass of individuals may not recover at all and die; $R$ can consist of individuals, who recover with temporary or permanent immunity, thereby further subdividing the epidemiological compartments. Using these notations, eight classes of compartmental models are possible - SI, SIS, SEI, SEIS, SIR, SIRS, SEIR and SEIRS . For example, in an SEIRS model, a fraction of the susceptible $(S)$ population gets exposed $(E)$ to infection, a part of which then becomes infectious $(I)$. Some from the $I$ class recover from the disease, and become part of the $R$ class with temporary immunity. When immunity is lost, they become susceptible to pathogen attack again, and enter the $S$ class. But in this paper we consider the standard SIR model where the individuals in the population are divided into three compartments. The susceptible $(S)$ which refers to the healthy individuals that has not yet come into contact with malaria disease. The infective $(I)$ are those who have become infected with malaria disease and are able to transmit the disease and the recovered $(R)$ are individuals who have recovered from malaria disease. The proportions of the individuals in the compartment of the population, i.e. S,I and $\mathrm{R}$, at time $\mathrm{t}$ is denoted as $\mathrm{S}(\mathrm{t}), \mathrm{I}(\mathrm{t})$ and $\mathrm{R}(\mathrm{t})$ respectively.

$\frac{\beta S I}{N} \gamma I$

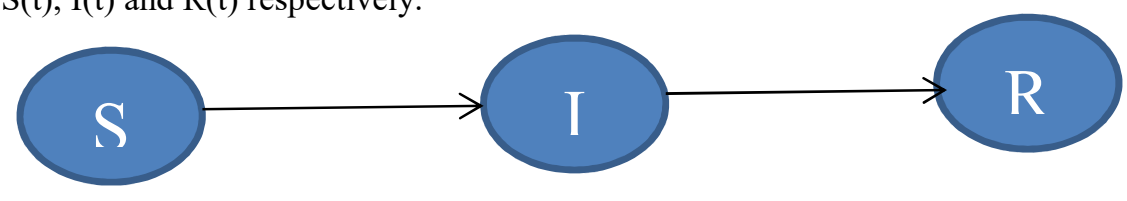

Figure 3 Flow chart showing the SIR model

$\beta=$ The per-capita contact rate between any two individuals.

$\frac{1}{N}=$ The proportion of infectious individuals in the population.

$\gamma=$ The per-capita rate of recovery once infectious.

$\gamma \times I=$ The rate at which infectious individuals are removed from the infectious class. 
$\beta \times S \times \frac{1}{N}=$ The rate at which susceptible individuals come across infectious individuals and then become infectious.

\subsection{Model description and formulation}

We formulate a mathematical model for the transmission and spread of malaria in the human and mosquito population. We formulate a model for the spread of malaria in the human and mosquito population with the total population size at time $\mathrm{t}$ given is by is given by $N_{H}(t)$ and $N_{v}(t)$ respectively. These are further compartmentalized into epidemiological classes shown below. The vector component of the model does not include immune class as mosquito never recover from infection, that is, their infective period ends with their death due to their relatively short life-cycle. Thus the immune class in the mosquito population is negligible and death occurs equally in all groups. Our model also excludes the immature mosquitoes since they do not participate in the infection cycle and are, thus, in the waiting period, which limits the vector population growth. Our model has the following variables and parameters:

$S_{H}(t)$ the number of susceptible human hosts at time t, $I_{H}(t)$ the number of infected human hosts at time $\mathrm{t}, R_{H}(t)$ the number of partially immune human hosts at time t, $S_{v}(t)$ the number of susceptible mosquito vectors at time $\mathrm{t}, I_{v}(t)$ the number of infected mosquito vectors at time $\mathrm{t}$, Given that the total number of bites made by the mosquitoes must equal the number of bites received by the humans, $\left(N_{H}, N_{V}\right)=\frac{N_{v}}{N_{H}}$ is a constant. Thus $m=\frac{N_{v}}{N_{H}}$ isnumber of female mosquitoes per human host.

Where $m=\frac{N_{v}}{N_{H}}$ is the female vector - host ratio, defined as the number of female mosquitoes perhuman host [2].

Our model, illustrated in Figure 3,seeks to look into the dynamics of the Susceptible-Infectious-Recovered and Susceptible Infectious (SIR-SI) malaria transmission, taking into account the three human classes, pre-school children ( $H_{1}$ - aged five years below), age from six to 25 ( $\mathrm{H}_{2} 5-25$ yearsold $)$ and the rest of the humans $\left(\mathrm{H}_{3}\right.$ older than twenty five years).

The human population $N_{H}$ is divided into nine compartments denoted by the following variables:

$S_{H 1}$ is the number of susceptible humans of age five (5) years and below;

$I_{H 1}$ is the number of infectious humans of age five (5) years and below;

$R_{H 1}$ is the number of recovered humans (with temporary immunity) of age five (5) years and below;

$S_{H 2}$ is the number of susceptible humans older than five (5) years and below 25 years old ;

$I_{H 2}$ is the number of infectious humans older than five (5) years and below 25 years old;

$R_{H 2}$ is the number of recovered humans (with temporary immunity) older than five (5) years and below 26 years old

$S_{H 3}$ is the number of susceptible humans of age greater than or equal to 25 years

$I_{H 3}$ is the number of infectious humans of age greater than or equal to 25 years

$R_{H 3}$ is the number of recovered humans of age greater than or equal to 25 years

In general, any member of the human population (pre-school or older) remains in the susceptible class $\mathrm{S}$ for a certain period unless bitten and becomes infectious. Once in the infective class I, an individual may recover without acquiring immunity and be transferred back into the susceptible class S, or stays in class I while infectious before moving to the recovered class R with an acquired temporary immunity. Humans in the recovered class may lose immunity and move back to the susceptible class.

Humans leave the population through natural death rate and through per-capita death rate due to infection. 
In particular, members of the susceptible pre-school children $S_{H 1}$ leave the population and move to infectious $I_{H 1}$ or grow older to susceptible $S_{H 2}$. Infectious $I_{H 1}$ leave the population and transfer to the infectious $I_{H 1}$, orrecovered $R_{H 1}$. For the recovered $R_{H 1}$ population, members may grow to the recovered $R_{H 2}$. Similarly, for the rest of the human population, susceptible $S_{H_{2}}$ members may move to the infectious class $I_{H_{2}}$. Infectious $I_{H_{2}}$ members can transfer to the recovered $R_{H_{2}}$. Recovered $R_{H_{2}}$ members can lose immunity and assume that not move back to the susceptible $S_{H_{2}}$ group.

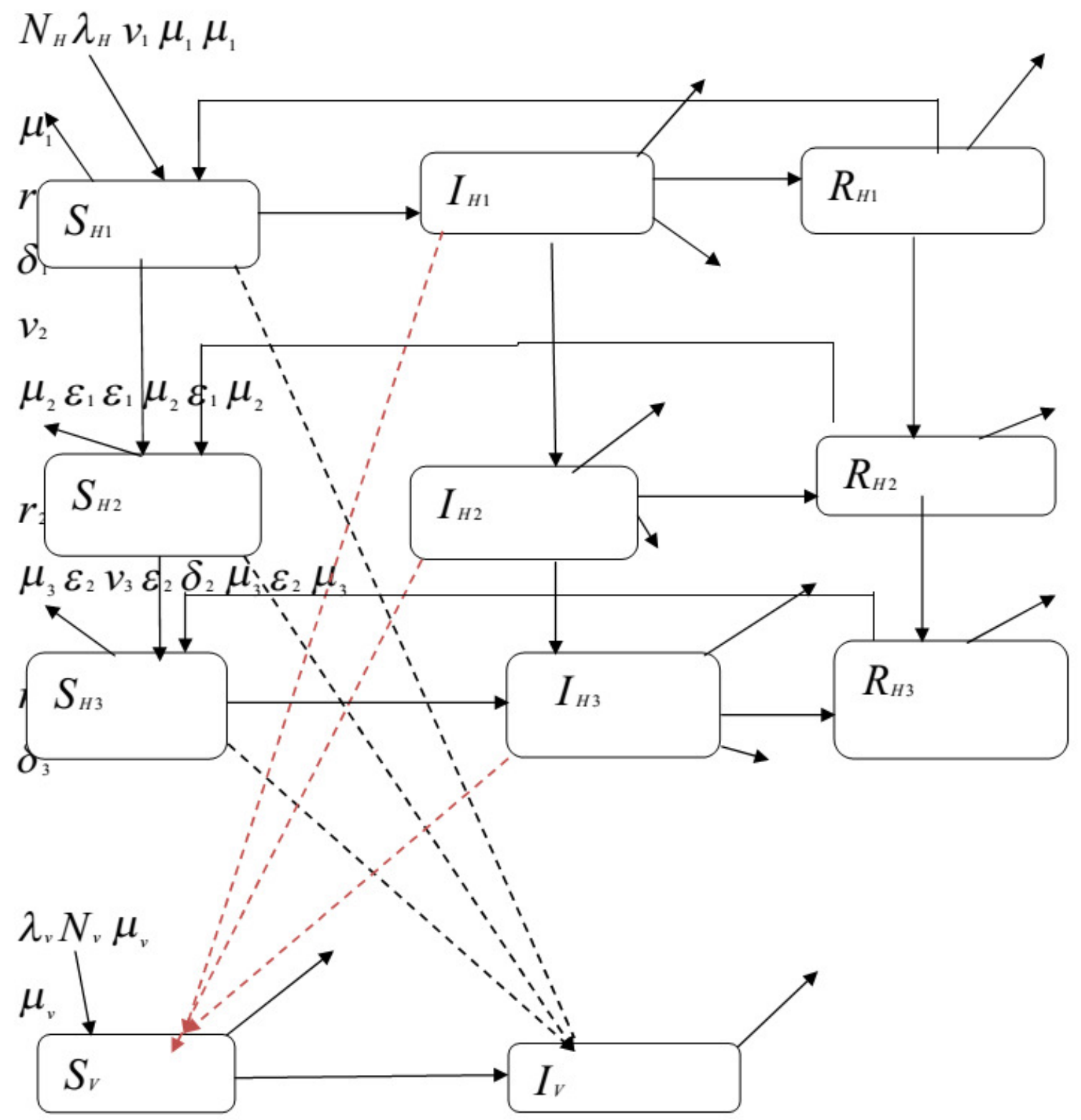

Figure 4: transmission pattern of SIR - SI with age-structured human population susceptible mosquitoes $S_{v}$ may transfer into the infective class $I_{v}$. Due to short life cycle of mosquitoes, the infective period ends with their death.

\subsection{Model Equations}

Applying the assumptions, definitions of variables and parameters and description of terms above, the differential equations which describe the dynamics of malaria in the human and mosquito populations are formulated as shown below: 
For first age group (0-5 years) we formulate:

$$
\begin{aligned}
& \frac{d S_{H 1}}{d t}=\lambda_{H} N_{H}-\mu_{1} S_{H 1}-\frac{a_{1}{ }^{b} S_{H 1} I_{V}}{N_{H}}+V_{1} R_{H 1}-\varepsilon_{1} S_{H 1}(3.5 .1) \\
& \frac{d I_{H 1}}{d t}=\frac{a_{1} b S_{H 1} I_{V}}{N_{H}}-\mu_{1} I_{H 1}-r_{1} I_{H 1}-\delta_{1} I_{H 1}-\varepsilon_{1} I_{H 1} \text { (3.5.2) } \\
& \frac{d R_{H 1}}{d t}= \\
& d t \quad-\mu_{1} R_{H 1}+r_{1} R_{H 1}-\varepsilon_{1} R_{H 1}-v_{1} R_{h 1} \text { (3.5.3) } \\
& \text { For second age group (5-25 years) we formulated } \\
& \frac{d S_{h 2}}{d t}=-\mu_{2} S_{h 2}-\frac{a_{2} b S_{H 2} I_{V}}{N_{H}}+V_{2} R_{H 2}+\varepsilon_{1} S_{H 1}-\varepsilon_{2} S_{H 2} \text { (3.5.4) } \\
& \frac{d I_{H 2}}{d t}=\frac{a_{2} b S_{H 2} I_{V}}{N_{H}}-\mu_{2} I_{H 2}-r_{2} I_{H 2}-\delta_{2} I_{H 2}+\varepsilon_{1} I_{H 1}-\varepsilon_{2} I_{H 2} \\
& \frac{d R_{H 2}}{d t}= \\
& -\mu_{2} R_{H 2}+r_{1} I_{H 2}+\varepsilon_{1} R_{H 1}-\varepsilon_{2} R_{H 2}-v_{2} R_{h 2}(3.5 .6)
\end{aligned}
$$

For the remaining age group (5-25 years) we formulate

$$
\begin{aligned}
& \frac{d S_{H 3}}{d t}=-\mu_{3} S_{h 3}-\frac{a_{3} b S_{H 3} I_{V}}{N_{H}}+V_{3} R_{H 3}+\varepsilon_{2} S_{H 2}(3.5 .7) \\
& \frac{d I_{H 3}}{d t}=\frac{a_{3} b S_{H 3} I_{V}}{N_{H}}-\mu_{3} I_{H 3}-r_{3} I_{H 3}-\delta_{3} I_{H 3}+\varepsilon_{2} I_{H 2}(3.5 .8) \\
& \frac{d R_{H 3}}{d t}=-\mu_{3} I_{H 3}+r_{3} I_{H 3}+\varepsilon_{2} I_{H 2}-v_{3} R_{h 3}(3.5 .9) \\
& \frac{d S_{V}}{d t}=\lambda_{V} N_{V}-\frac{a_{1} C S_{V} I_{H 1}}{N_{H}}-\frac{a_{2} c S_{v} I_{H 2}}{N_{H}}-\frac{a_{3} c S_{v} I_{H 3}}{N_{H}}-\mu_{V} S_{V} \\
& \frac{d I_{V}}{d t}= \\
& a_{1} C S_{V} I_{H 1}+\frac{a_{2} c S_{v} I_{H 2}}{N_{H}}+\frac{a_{3} c S_{v} I_{H 3}}{N_{H}}-\mu_{V} S_{V}(3.5 .11)
\end{aligned}
$$

The total population size $N_{H 1}, N_{H 2}, N_{H 3}$ and $N_{v}$ can be determined by

$N_{H 1}=S_{H 1}+I_{H 1}+R_{H 1}=1$

$N_{H 2}=S_{H 2}+I_{H 2}+R_{H 2}=1$

$N_{H 3}=S_{H 3}+I_{H 3}+R_{H 3}=1$

The total population of the first age group is derived by adding equations (3.5.1)-(3.5.3) to obtain $\frac{d N_{H 1}}{d t}=\lambda_{H} N_{H 1}-\left(\mu_{1}+\varepsilon_{1}\right) S_{H 1}-\left(\mu_{1}+\varepsilon_{1}+\delta_{1}\right) I_{H 1}-\left(\mu_{1}+\varepsilon_{1}\right) R_{H 1}$

The total population of the second age group is derived by adding equations (3.5.4)-(3.5.6) to obtain $\frac{d N_{H 2}}{d t}=\left(\mu_{2}+\varepsilon_{2}\right) S_{H 2}-\left(\mu_{2}+\varepsilon_{2}+\delta_{2}\right) I_{H 2}-\left(\mu_{2}+\varepsilon_{2}\right) R_{H 2}+\varepsilon_{1}\left(S_{H 1}+I_{H 1}+R_{H 1}\right)$ 
And the total population of the second age group is derived by adding equations (3.5.7)-(3.5.9) to obtain

$$
\frac{d N_{H 3}}{d t}=-\mu_{3} S_{H 3}-\left(\mu_{3}+\delta_{3}\right) I_{H 3}+\varepsilon_{2}\left(S_{H 2}+I_{H 2}+R_{H 2}\right)-\mu_{3} R_{H 3}
$$

In the model, the term $\frac{a_{i} b S_{H 1} I_{v}}{N_{H}}$ denotes the rate at which the human hosts $S_{H 1}$ get infected by infected mosquitoes $I_{v}$ for $i=1,2,3$ and $\frac{a_{j} c S_{v} I_{H}}{N_{H}}$ refers to the rate at which the susceptible mosquitoes $S_{v}$ are infected by the infected human hosts $I_{H}$ for $j=1,2,3$.It is important to note that the rate of infection of human host $S_{H}$ by infected vector $I_{v}$ is dependent on the total number of humans $N_{H}$ available per infected vector [19] and[36]. The model assumes that all newborns are susceptible in both human and mosquito populations.

The per capita birth rate for humans and mosquito populations, $\lambda_{H}$ and $\lambda_{v}$ respectively, are both positive. The human and mosquito populations experience per capita natural death rate $\mu_{1}, \mu_{2}, \mu_{3}$ and $\mu_{v}$ respectively. Infected humans die due to infection at per capita death rate $\delta_{1}, \delta_{2}$ and $\delta_{3}$ respectively. We can consider recovered human individuals lose their immunity at per capital rates. To ensure that we have a stable positive human population, we assume that $\lambda_{H}>\delta_{1}+\delta_{2}+\delta_{3}+\mu_{1}+\mu_{2}+\mu_{3}$.

Moreover, it is clear that the birth rate should be greater than the per capita rate $\varepsilon_{1}$ and $\varepsilon_{2}$ of growing up.

Consider the interaction between the two populations shown in Figure 3. If $a_{1}$ is the average number of bites per mosquito per unit time, then there are $\frac{a_{1} N_{v}}{N_{H}}$ bites per human per time. For the pre-school humans $H_{1}$, note that there are $S_{H 1}$ susceptible humans and the proportion of the total number of bites that are possibly infectious to humans is $\frac{I_{v}}{N_{v}}$. Then the number of potentially infectious bites given to susceptible pre-school children is $\frac{a_{1} I_{v} S_{H 1}}{N_{H}}$ bites per time. However, only a fraction of these bites, namely $b$, successfully infect the pre-school children. Hence we have:

$$
\frac{(\text { pre-schoolhumansinfected) }}{\text { (unittime) }}=\frac{a_{1} b I_{v} S_{H 1}}{N_{H}}
$$

The same formulation is constructed for the rest of the human group $H_{2}$ and $H_{3}$. Similarly for the mosquito population, we have

$$
\frac{\text { (mosquitoes infected) }}{\text { (unittime) }}=\frac{\left(a_{1} c I_{H 1} s_{V}\right)}{N_{H}}+\frac{\left(a_{2} c I_{h 2} S_{v}\right)}{N_{H}}+\frac{a_{3} c s_{v} I_{h 3}}{N_{H}}
$$

To analyze the age-structured malaria model given by Equations (3.5.1)-(3.5.11), we construct the fractional quantities by scaling the population of each class by the total species population (We normalize our dynamical equations by letting). The proportions for the system are $S_{h 1}=\frac{S_{H 1}}{N_{H}}, S_{h 2}=\frac{S_{H 2}}{N_{H}}, S_{h 3}=\frac{S_{H 3}}{N_{H}}, i_{h 1}=\frac{I_{H 1}}{N_{H}}$, $i_{h 2}=\frac{I_{H 2}}{N_{H}}, i_{h 3}=\frac{I_{H 3}}{N_{H}}, r_{h 1}=\frac{R_{H 1}}{N_{H}}, r_{h 2}=\frac{R_{H 2}}{N_{H}}, r_{h 3}=\frac{R_{H 3}}{N_{H}}, S_{v}=\frac{S_{V}}{N_{V}}$ and $i_{v}=\frac{I_{V}}{N_{V}}$

Let $r_{h 1}=1-\left(i_{h 1}-S_{h 1}\right)$

$r_{h 2}=1-\left(i_{h 2}-s_{h 2}\right)$

$r_{h 3}=1-\left(i_{h 3}-s_{h 3}\right)$

Differentiating the scaling equations (3.5.1)-(3.5.11) and solving for the derivatives of the scaledvariables, we obtain the following reduced system of nine DE 


$$
\begin{aligned}
& \frac{d i_{h 1}}{d t}=\frac{1}{N_{H}}\left(\frac{d I_{H 1}}{d t}-i_{h 1} \frac{d N_{H}}{d t}\right) \\
& \frac{d s_{h 1}}{d t}=\frac{1}{N_{H 1}}\left(\frac{d s_{H 1}}{d t}-s_{h 1} \frac{d N_{H 1}}{d t}\right) \quad=a b m s_{h 1} i_{v}-\left(r_{1}+\delta_{1}+\lambda_{h}\right) i_{h 1}+\delta_{1} i_{h 1}^{2}=0 \\
& \frac{d i_{v}}{d t}=\frac{1}{N_{v}}\left(\frac{d I_{v}}{d t}-i_{v} \frac{d N_{v}}{d t}\right)=\lambda_{h}\left(1-s_{h 1}\right)-a b m s_{h 1} i_{v}+v_{1}\left(1-s_{h 1}-i_{h 1}\right)+\delta_{1} s_{h 1} i_{h 1}=0 \\
& \frac{d i_{h 2}}{d t}=\frac{1}{N_{H 2}}\left(\frac{d I_{H 2}}{d t}-i_{h 2} \frac{d N_{H 2}}{d t}\right) \\
& =a_{21}-\lambda_{v} i_{v}=0(3.5 .14)
\end{aligned}
$$

\subsection{Existence and Stability of Equilibrium Points}

3.3.1. Equilibrium points of the model

The equilibrium analysis helps to achieve this. Thus, we shall consider two equilibriums the diseases-free equilibrium points and endemic equilibrium points. The points at which the differential equations of the system (3.5.12)-(3.5.20) equal to zero are referred to as equilibrium points or steady-state solutions. It is important to note that there are no trivial equilibrium points as long as the recruitment terms $N_{H 1}, N_{H 2}, N_{H 3}$ and $N_{v 1}, N_{v 2}, N_{v 3}$ are not zero.

\subsubsection{Existence of Disease-free Equilibrium Point}

Disease-free equilibrium points are steady-state solutions where there is no malaria infection (no disease). We define the "diseased" classes as the human or mosquito populations that are either infectious, or recovered, that is $i_{h 1}, i_{h 1}, i_{h 3}, i_{v}=0$.

Thus, the disease-free equilibrium point, $E_{0}$, for the malaria model (3.5.12)-(3.5.14)in the absence of disease implies that $i_{h 1}=i_{v}=0$.

From equation (3.5.12)- (3.5.14) at $i_{h 1}=i_{v}=0$ we have

$$
\lambda_{h}\left(1-S_{h 1}\right)-+v_{1}\left(1-S_{h 1}\right)=0
$$


$\Rightarrow \lambda_{h}-\lambda_{h} S_{h 1}+v_{1}\left(1-S_{h 1}\right)=0$

$\Rightarrow S_{h 1}=\frac{v_{1}+\lambda_{h}}{v_{1}+\lambda_{h}}=1$

This implies that the disease free equilibrium point is given by $\left(s_{h 1}{ }^{*}, i_{h 1}, i_{v 1}\right)=(1,0,0)$

\subsubsection{Calculating Reproductive Number $R_{0}$ for the first age group}

To calculate $R_{0}$, we use the compartmental model approach presented by VandenDriessche and Watmough and Diekmann et al [12].

$F=\left[\frac{\partial F_{i}\left(x_{0}\right)}{\partial x_{j}}\right]$ and $v=\left[\frac{\partial v_{i}\left(x_{0}\right)}{\partial x_{j}}\right]$

where $F_{i}=$ be the rate of appearance of new infections in compartments $\mathrm{i}$

$v_{i}=$ be the transfer of individuals out of compartments i by all other means

$v_{i}^{-}=$be the rate transfer of individuals out of compartment $\mathrm{i}$

$v_{i}^{+}=$be the rate transfer of individuals in to compartment $\mathrm{i}$ by all other means

$x_{0}=$ be the disease free equilibrium

$R_{0}=$ is the largest eigenvalues of Matrix of partial derivatives

Then $F v^{-1}=\left[\frac{\partial F_{i}\left(x_{0}\right)}{\partial x_{j}}\right] \times\left[\frac{\partial v_{i}\left(x_{0}\right)}{\partial x_{j}}\right]$

To find reproduction number $R_{0}$ for the first age group will refer to the ODEs (3.5.12)-(3.5.14) and it is important to distinguish new infections from all other changes in population

Gains terms Loss terms

$F_{1}=a_{1} b m i_{v} s_{h 1} v_{1}=\left(r_{1}+\delta_{1}+\lambda_{h}\right) i_{h 1}+\delta_{1} i_{h 1}^{2}$

$F_{2}=0 v_{2}=\lambda_{h}\left(1-s_{h 1}\right)+a_{1} b m i_{v} s_{h 1}+v_{1}\left(1-s_{h 1}-i_{h 1}\right)+\delta_{1} s_{h 1} i_{h 1}$

$F_{3}=a c\left(1-i_{v}\right) v_{3}=\lambda_{v} i_{v}$

Then the Jacobian matrix of $\mathrm{F}$ (gain terms) is given by

$$
F\left(i_{h 1} *, s_{h 1} *, i_{v} *\right)=\left[\begin{array}{ccc}
0 & a_{1} b m i_{v} & a_{1} b m s_{h 1} \\
0 & 0 & 0 \\
c a_{1}\left(1-i_{v}\right) & 0 & -a_{1} c i_{h 1}
\end{array}\right]
$$

Then the Jacobian of $\mathrm{F}$ at equilibrium point $E_{0}=\left(s_{h 1}{ }^{*}, i_{h 1}{ }^{*}, i_{v}{ }^{*}\right)=(1,0,0)$ is given by

$$
F(0,1,0)=\left[\begin{array}{ccc}
0 & 0 & a b m s_{h 1} \\
0 & 0 & 0 \\
c a_{1} & 0 & 0
\end{array}\right]
$$

And the Jacobian matrix of $\mathrm{V}$ (loss terms) is given by

$$
\begin{gathered}
v\left(i_{h 1}{ }^{*}, s_{h 1}{ }^{*}, i_{v}^{*}\right)=\left[\begin{array}{ccc}
k_{1} & 0 & 0 \\
k_{2} & k_{3} & k_{4} \\
0 & 0 & \lambda_{v}
\end{array}\right] \\
\text { where } \\
k_{1}=r_{1}+\delta_{1}+\lambda_{h}+2 \delta_{1} i_{h 1}
\end{gathered}
$$




$$
\begin{gathered}
k_{2}=v_{1}+\delta_{1} s_{h 1} \\
k_{3}=v_{1}+\lambda_{h}+\delta_{1} i_{h 1}+a_{1} b m i_{v} \\
k_{4}=a_{1} b m s_{h 1}
\end{gathered}
$$

Then the Jacobian matrix of loss terms $\mathrm{V}$ at $\operatorname{DFE}(0,1,0)$ is given by

$$
\begin{gathered}
v(0,1,0)=\left[\begin{array}{ccc}
k_{11} & 0 & 0 \\
k_{22} & k_{33} & k_{44} \\
0 & 0 & \lambda_{v}
\end{array}\right] \\
\text { where } k_{11}=r_{1}+\delta_{1}+\lambda_{h}, k_{22}=v_{1}+\delta_{1}, k_{33}=v_{1}+\lambda_{h}, k_{44}=a_{1} b m
\end{gathered}
$$

The matrix $F v^{-1}$ is called the next generation matrix of the epidemiological model.

$$
\text { To find } \begin{aligned}
v^{-1} \text { let } \mathrm{A} & =\left(\begin{array}{lll}
a & b & c \\
d & e & f \\
g & h & i
\end{array}\right) \text {. Then }\left(\begin{array}{lll}
a & b & c \\
d & e & f \\
g & h & i
\end{array}\right) \times\left[\begin{array}{ccc}
k_{11} & 0 & 0 \\
k_{22} & k_{33} & k_{44} \\
0 & 0 & \lambda_{v}
\end{array}\right]=\left(\begin{array}{lll}
1 & 0 & 0 \\
0 & 1 & 0 \\
0 & 0 & 1
\end{array}\right) \\
& \left(\begin{array}{lll}
a k 11+b k 22 & b k 33 & b k 44+c h v \\
d k 11+e k 22 & e k 33 & e k 44+f h v \\
g k 11+e k 22 & h k 33 & h k 44+i h v
\end{array}\right)=\left(\begin{array}{lll}
1 & 0 & 0 \\
0 & 1 & 0 \\
0 & 0 & 1
\end{array}\right) \\
& \Rightarrow\left\{\begin{array}{c}
a k 11+b k 22=1 \\
b k 33=0 \\
b k 44+c h v=0
\end{array} \Rightarrow b=c=0 \text { and } a=\frac{1}{k 11}\right.
\end{aligned}
$$

$$
\begin{gathered}
\left\{\begin{array} { c } 
{ d k 1 1 + e k 2 2 = 0 } \\
{ e k 3 3 = 1 } \\
{ e k 4 4 + f h v = 0 }
\end{array} \left\{d=\frac{-k 22}{k 11 k 33}, f=\frac{-k 44}{k 33 h v} \text { and } e=\frac{1}{k 33}\right.\right. \\
\begin{array}{c}
g k 11+h k 22=0 \\
h k 33=0 \\
h k 44+i h v=1
\end{array} \\
\Rightarrow\left(\begin{array}{ccc}
\frac{1}{k 11} & 0 & 0 \\
\frac{-k 22}{k 11 k 33} & \frac{1}{k 33} & \frac{-k 44}{k 33 h v} \\
0 & 0 & \frac{1}{h v}
\end{array}\right) \\
\text { Hence } F v^{-1}=\left(\begin{array}{ccc}
0 & 0 & a_{1} b m s_{h 1} \\
0 & 0 & 0 \\
c a_{1} & 0 & 0
\end{array}\right] \times\left(\begin{array}{ccc}
\frac{1}{k 11} & 0 & 0 \\
\frac{-k 22}{k 11 k 33} & \frac{1}{k 33} & \frac{-k 44}{k 33 h v} \\
0 & 0 & \frac{1}{h v}
\end{array}\right)
\end{gathered}
$$




$$
=\left(\begin{array}{ccc}
0 & 0 & \frac{a_{1} b m s_{h 1}}{\lambda_{v}} \\
0 & 0 & 0 \\
\frac{c a_{1}}{k_{11}} & 0 & 0
\end{array}\right)
$$

Then the spectral radius (the dominant eigen value) $F v^{-1}$ is equal to $R_{0}$.

$$
\begin{gathered}
\text { That is }|J-\lambda I|=0 \\
\left|\begin{array}{ccc}
\lambda & 0 & \frac{a_{1} b m}{\lambda_{v}} \\
0 & -\lambda & 0 \\
\frac{c a_{1}}{k_{11}} & 0 & -\lambda
\end{array}\right|=0
\end{gathered}
$$

which forms the characteristics equation

$$
\begin{aligned}
& \Rightarrow-\lambda^{3}+\frac{a_{1}^{2} b c m \lambda}{k_{11} \lambda_{v}}=0 \\
& =-\lambda\left(\lambda^{2}-\frac{a_{1}^{2} b c m}{k_{11} \lambda_{v}}\right)=0 \\
& \Rightarrow \lambda=0 \text { or } \lambda^{2}=\frac{a_{1}^{2} b m c}{k_{11} \lambda_{v}}
\end{aligned}
$$

Therefore the dominant Eigen values of $F V^{-1}$ is $\lambda^{2}=\frac{a_{1}^{2} b m c}{k_{11} \lambda_{v}}$

$$
\Rightarrow R_{0}^{2}=\frac{a_{1}^{2} b m c}{k_{11} \lambda_{v}} \text {, then } R_{0}=\sqrt{\frac{a_{1}^{2} b m c}{k_{11} \lambda_{v}}}(3.5 .21)
$$

This is the required reproduction number for the first age group.

From (3.5.21) if $a_{1}^{2} b c m>k 11 h v$ then $R_{0}>1$, this means that the equilibrium point is unstable (i.e the malaria disease is highly persistence to the population). If $a_{1}^{2} b c m<k 11 h v$ then $R_{0}<1$ and the equilibrium point is stable (i.e the disease is die out).

\subsubsection{Equilibrium points and stability analysis}

To determine the stability of the equilibrium points of the given model we evaluate the steady state of the given system with following conditions.

$>$ Disease free equilibrium points (in the absence of infection) $\boldsymbol{i}_{h 1}, \boldsymbol{i}_{v 1}=0$

$>$ Endemic equilibrium points, that is $i_{h 1}, \boldsymbol{i}_{v 1} \neq 0$

\subsubsection{Local Stability analysis $(\mathrm{sh} 1 *$,ih $1 *$,iv $1 *)=(0,1,0)$}

To determine the stability of the system, we will consider linearizing the systems of equations (3.5.12)- (3.5.14) about the equilibrium points by taking the Jacobian of them.

The Jacobian matrix at any point $\left(i_{h 1} *, s_{h 1} * i_{v} *\right)$ is given by

$$
J\left(i_{h 1}^{*}, s_{h 1} * i_{v}^{*}\right)=\left[\begin{array}{ccc}
-\left(\lambda_{h}+r_{1}+\delta_{1}\right)+2 \delta_{1} i_{h 1} & -a_{1} b m i_{v}^{*} & a_{1} b m S_{h 1}^{*} \\
-v_{1}+\delta_{1} s_{h 1} * & -\left(\lambda_{h}+v_{1}+a_{1} b m i_{v}-\delta_{1} i_{h 1}\right) & -a_{1} b m S_{h 1} * \\
a_{1} c\left(1-i_{v}\right) & 0 & -a_{1} c i_{h 1}-\lambda_{v}
\end{array}\right]
$$

For the disease-free equilibrium, we evaluate the Jacobian matrix at the equilibrium points $\left(i_{h 1} *, s_{h 1} * i_{v} *\right)=(0,1,0)$ and hence we obtain 


$$
J(0,1,0)=\left[\begin{array}{ccc}
-\left(\lambda_{h}+r_{1}+\delta_{1}\right) & 0 & a_{1} b m \\
-v_{1}+\delta_{1} & -\left(\lambda_{h}+v_{1}\right) & -a_{1} b m \\
a_{1} c & 0 & -\lambda_{v}
\end{array}\right]
$$

The characteristic polynomial of $(3.5 .22)$ is

$$
J(0,1,0)=\left[\begin{array}{ccc}
-\left(\lambda_{h}+r_{1}+\delta_{1}\right)-\lambda & 0 & a_{1} b m \\
-v_{1}+\delta_{1} & -\left(\lambda_{h}+v_{1}\right)-\lambda & -a_{1} b m \\
a_{1} c & 0 & -\lambda_{v}-\lambda
\end{array}\right]
$$

Now applying $\left|J-\lambda I_{3}\right|=0$ on (ii) we have

$$
\left|\begin{array}{ccc}
-\left(\lambda_{h}+r_{1}+\delta_{1}\right)-\lambda & 0 & a_{1} b m \\
-v_{1}+\delta_{1} & -\left(\lambda_{h}+v_{1}\right)-\lambda & -a_{1} b m \\
a_{1} c & 0 & -\lambda_{v}-\lambda
\end{array}\right|=0 \text { (3.5.24) }
$$

Solving for roots of the characteristic polynomial (with real coefficient) given in the Jacobian matrix leads to the characteristic equation

$\lambda^{3}+k_{1} \lambda^{2}+k_{2} \lambda+k_{3}=0(3.5 .25)$

Where $k 1, k 2$, and $k 3$ are the coefficients given by the expressions: $k_{3}=-\left(\lambda_{v}+v_{1}\right) a_{1}^{2} b c m+\delta_{1} v_{1} \lambda_{v}+\lambda_{h}^{2} \lambda_{v}+r_{1} v_{1} \lambda_{v}+r_{1} \lambda_{h} \lambda_{v}+\delta_{1} \lambda_{h} \lambda_{v}+\lambda_{v} v_{1} \lambda_{h}$ $k_{2}=\left(\delta_{1}+r_{1}+\lambda_{h}\right) \lambda_{v}-a_{1}^{2} b c m+\delta_{1} \lambda_{h}+r_{1} v_{1}+\lambda_{h}^{2}+\delta_{1} v_{1}+\lambda_{v} v_{1}+\lambda_{h} \lambda_{v}+r_{1} \lambda_{h}+\lambda_{h} \lambda_{v}$ $=\left(\frac{1}{R_{0}^{2}}-1\right)+\left(\delta_{1}+r_{1}+\lambda_{h}\right) \lambda_{h}+\left(r_{1}+\delta_{1}+\lambda_{v}\right) \mathcal{v}_{1}+2 \lambda_{h} \lambda_{v}$ $k_{1}=v_{1}+\lambda_{v}+2 \lambda_{h}+\delta_{1}+r_{1}$

Using Routh-Hurwitz criteria (Edelstein-Keshet 1988), the equilibrium is locally stable if the following conditions are satisfied.

Theconditions are:

(i) $k_{1}>0$

(ii) $k_{3}>0$

(iii) $k_{1} k_{2}-k_{3}>0$

If these conditions are true, then all roots of the characteristic polynomial equation have negative real part, which concludes that there is a stable equilibrium. To show the three condition (i),(ii) and (iii) let:

(i) $k_{1}>0$

Clearly $k_{1}>0$ since all parameters are positive.

(ii) $k_{3}>0$

$$
\begin{aligned}
& k_{3}=\delta_{1} v_{1} \lambda_{v}+\lambda_{v} \lambda_{h}^{2}+r_{1} v_{1} \lambda_{v}+r_{1} \lambda_{v} \lambda_{h}+\delta_{1} \lambda_{h} \lambda_{v}+\lambda_{h} v_{1} \lambda_{v}-a_{1}^{2} b c m\left(\lambda_{v}+v_{1}\right) \\
& \quad=\left(\left(\delta_{1}+r_{1}+\lambda_{h}\right) \lambda_{v}-a_{1}^{2} b c m\right) v_{1}+\left(\delta_{1}+r_{1}+\lambda_{h}\right) \lambda_{v} \lambda_{h}-a_{1}^{2} b c m \lambda_{h} \\
& =\left(\frac{1}{R_{0}^{2}}-1\right) v_{1}+\left(\delta_{1}+r_{1}+\lambda_{h}\right) \lambda_{v} \lambda_{h}-a_{1}^{2} b c m \lambda_{h} \\
& =\left(\frac{1}{R_{0}^{2}}-1\right) v_{1}+\left(\frac{1}{R_{0}^{2}}-1\right) \lambda_{h} \\
& =\left(\frac{1}{R_{0}^{2}}-1\right)\left(\lambda_{h}+v_{1}\right)
\end{aligned}
$$


Clearly the disease free equilibrium point is locally asymptotically stable if $R_{0}^{2}<1$. If $R_{0}^{2}>1$ then, $k_{3}<0$. Hence, there is only one sign change in the sequence, $k_{1}, k_{2}, k_{3}$ and so by Descartes rule of sign, there is one eigenvalue with positive real part. This implies that the disease-free equilibrium point $(0,1,0)$ is unstable.

$\Rightarrow k_{3}>0{ }_{\text {if }} R_{0}^{2}<1$ and $k_{3}<0 R_{0}^{2}>1$

(iii) $k_{1} k_{2}-k_{3}>0$

$\Rightarrow\left(\left(\frac{1}{R_{0}^{2}}-1\right)+\left(\delta_{1}+r_{1}+\lambda_{h}\right) \lambda_{h}+\left(r_{1}+\delta_{1}+\lambda_{v}\right) \nu_{1}+2 \lambda_{h} \lambda_{v}\right)\left(v_{1}+\lambda_{v}+2 \lambda_{h}+\delta_{1}+r_{1}\right)-$ $\left(\frac{1}{R_{0}^{2}}-1\right)\left(\lambda_{h}+v_{1}\right)$

Hence the DFE point $(0,1,0)$ are unstable by Routh-Hurwitz stability criteria. But if $R_{0}<1$ the DFE point $(0,1,0)$ are stable.

\subsubsection{Existence and uniqueness of endemic equilibrium (ih1**,sh $1 * *$,iv1 $1 *$ )}

Here, the condition for the existence and uniqueness of the endemic equilibrium of model system Eq. (3.5.12)(3.5.14) is determined. Let $\mathrm{X}^{*}=\left(\mathrm{ih} 1^{*}, \mathrm{sh} 1^{*}, \mathrm{iv} 1^{*}\right)$ be the endemic equilibrium. To find the endemic equilibrium, we equate all equations in model system Eq. (3.5.12)-(3.5.14) to zero and rewrite it as follows:

$a_{1} b m S_{h 1} i_{v}-\left(r_{1}+\delta_{1}+\lambda_{h}\right) i_{h 1}+\delta_{1} i_{h 1}^{2}=0$

$\lambda_{h}\left(1-S_{h 1}\right)-a_{1} b m S_{h 1} i_{v}+v_{1}\left(1-S_{h 1}-i_{h 1}\right)+\delta_{1} S_{h 1} i_{h 1}=0$

$c a_{1}\left(1-i_{v 1}\right) i_{h 1}-\lambda_{v} i_{v 1}=0$

In the presence of infection (ih1,iv1 \# 0), the mathematical model (3.5.12)-(3.5.14) has a unique endemic equilibrium given by $\mathrm{X}^{*}=\left(\mathrm{ih} 1^{*}, \mathrm{sh} 1^{*}, \mathrm{iv} 1^{*}\right)$ which are determined from the system as follows. The associated Jacobian matrix is obtained as:

$$
J\left(i_{h 1}, S_{h 1}, i_{v}\right)=\left[\begin{array}{ccc}
-\left(\lambda_{h}+r_{1}+\delta_{1}\right)+2 \delta_{1} i_{h 1} & -a_{1} b m i_{v} & a_{1} b m S_{h 1} \\
-v_{1}+\delta_{1} s_{h 1} & -\left(\lambda_{h}+v_{1}+a_{1} b m i_{v}-\delta_{1} i_{h 1}\right) & -a_{1} b m S_{h 1} \\
a_{1} c\left(1-i_{v}\right) & 0 & -a_{1} c i_{h 1}-\lambda_{v}
\end{array}\right]
$$

Then the Jacobian matrix of above matrix at $\operatorname{EEP}\left(i_{h 1} *, s_{h 1} * i_{v} *\right)$ is given by

$$
J\left(i_{h 1} *, s_{h 1} * i_{v} *\right)=\left[\begin{array}{ccc}
-\left(\lambda_{h}+r_{1}+\delta_{1}\right)+2 \delta_{1} i_{h 1} * & -a_{1} b m i_{v}^{*} & a_{1} b m \boldsymbol{S}_{h 1}^{*} \\
-v_{1}+\delta_{1} s_{h 1} * & -\left(\lambda_{h}+v_{1}+a_{1} b m i_{v}^{*}-\delta_{1} i_{h 1}^{*}\right) & -a_{1} b m \boldsymbol{S}_{h 1}^{*} \\
a_{1} c\left(1-i_{v} *\right) & 0 & -a_{1} c i_{h 1} *-\lambda_{v}
\end{array}\right]
$$

Obtaining the eigenvalues as usual from of the formula $\left|J\left(H^{*}-\lambda I\right)\right|=0$

$$
\Rightarrow\left|\begin{array}{ccc}
-\left(\lambda_{h}+r_{1}+\delta_{1}\right)+2 \delta_{1} i_{h 1}-\lambda & -a_{1} b m i_{v} * & a_{1} b m S_{h 1}^{*} \\
-v_{1}+\delta_{1} s_{h 1} * & -\left(\lambda_{h}+v_{1}+a_{1} b m i_{v}-\delta_{1} i_{h 1}\right)-\lambda & -a_{1} b m S_{h 1}^{*} \\
a_{1} c\left(1-i_{v}\right) & 0 & -a_{1} c i_{h 1}-\lambda_{v}-\lambda
\end{array}\right|=0
$$

Since the model is not expressed explicitly then we use Matlab or Mathematica and the results discussed in chapter four:

\subsubsection{Calculating Reproductive Number $R_{01}$ for the second age group}

To calculate the reproductive number of age group two we consider equation (3.5.15)-(3.5.17).

$$
\begin{gathered}
a_{2}{ }^{b m} S_{h 2} i_{v}-\left(r_{2}+\delta_{2}-\varepsilon_{1}\right) i_{h 2}-\varepsilon_{1} i h_{1}+\delta_{2} i_{h 2}^{2}=0(\mathrm{i}) \\
-a_{2}{ }^{b m} S_{h 2} i_{v 2}-\varepsilon_{1} S_{h 1}+\delta_{2} S_{h 2} i_{h 2}-\varepsilon_{2} S_{h 2}+v_{2}\left(1-S_{h 2}-i_{h 2}\right)=0(\mathrm{ii}) \\
c a_{2}\left(1-i_{v 2}\right) i_{h 2}-\lambda_{v} i_{v 2}=0
\end{gathered}
$$


In the absence of infection (ih2 $=\mathrm{iv} 2=0$ ) equation (i)-(iii) becomes

$$
\begin{gathered}
\mathcal{E}_{1} S_{h 1}-\varepsilon_{1} S_{h 2}+v_{2}\left(1-S_{h 2}\right)=0 \\
\Rightarrow \varepsilon_{1} S_{h 1}-S_{h 2}\left(\varepsilon_{1}+v_{2}\right)+v_{2}=0 \\
\Rightarrow S_{h 2}=\frac{\varepsilon_{1} S_{h 1}+v_{2}}{v_{2}+\varepsilon_{1}} \\
=\frac{\varepsilon_{1}+v_{2}}{v_{2}+\varepsilon_{1}}=1
\end{gathered}
$$

To find reproduction number $R_{0}$ for the second age group will refer to the ODEs (3.5.15)-(3.5.17) it is important to distinguish new infections from all other changes in population.

$$
\begin{gathered}
\text { Gains terms } \\
F_{1}=a_{2} b m i_{v} F_{2}=0 F_{3}=c a_{2}\left(1-i_{v}\right) i_{h 2} \\
\text { Loss terms } \\
v_{1}=\left(r_{2}+\delta_{2}-\varepsilon_{1}\right) i_{h 2}+\delta_{1} i_{h 2}^{2}-\varepsilon_{1} i_{h 1} \\
v_{2}=a_{2} b m s_{h 2} i_{v}+v_{2}\left(1-s_{h 2}-i_{h 2}\right)+\delta_{2} s_{h 2} i_{h 2}+\varepsilon_{1} s_{h 1}+\varepsilon_{1} s_{h 2} \\
v_{3}=\lambda_{v} i_{v}
\end{gathered}
$$

Then the Jacobian matrix of $\mathrm{F}$ (gain terms) is given by

$$
F\left(i_{h 2}, s_{h 2}, i_{v}\right)=\left[\begin{array}{ccc}
0 & a_{2} b m i_{v} & a_{2} b m s_{h 2} \\
0 & 0 & 0 \\
a_{2} c\left(1-i_{v}\right) & 0 & -a_{2} c i_{h 2}
\end{array}\right]
$$

Then the Jacobian of $\mathrm{F}$ at equilibrium point $E_{0}=\left(i_{h 2}{ }^{*}, S_{h 2}{ }^{*}, i_{v 2}{ }^{*}\right)=(0,1,0)$ is given by

$$
\begin{gathered}
F(0,1,0)=\left[\begin{array}{ccc}
0 & 0 & a_{2} b m \\
0 & 0 & 0 \\
c a_{2} & 0 & 0
\end{array}\right] \\
\text { And the Jacobian matrix of } \\
v\left(i_{h 2}, s_{h 2}, i_{v}\right)=\left[\begin{array}{ccc}
w_{1} & 0 & 0 \\
w_{2} & w_{3} & w_{4} \\
0 & 0 & \lambda_{v}
\end{array}\right] \\
\text { where } w_{1}=\delta_{2}+r_{2}-\varepsilon_{1}+2 \delta_{2} i_{h 2} \\
w_{2}=v_{2}+\delta_{2} s_{h 2} \\
w 3=a_{2} b m i_{v}+v_{2}+\delta_{2} i_{h 2} \\
w_{4}=a_{2} b m s_{h 2}
\end{gathered}
$$

And the Jacobian matrix of $\mathrm{v}$ (loss terms) is given by

Then theJacobian matrix of loss terms $v$ at $\operatorname{DFE}(0,1,0)$ is given by

$$
V(0,1,0)=\left[\begin{array}{ccc}
w_{11} & 0 & 0 \\
w_{22} & w_{33} & w_{44} \\
0 & 0 & \lambda_{v}
\end{array}\right]
$$

Where: $w_{11}=\delta_{2}+r_{2}-\varepsilon_{1} \& w_{22}=v_{2}+\delta_{2}, w_{33}=v_{2}+\varepsilon_{1}, w_{44}=a_{2} b m$

The matrix $F v^{-1}$ is called the next generation matrix of the epidemiological model. 


$$
\begin{aligned}
& \text { To find } v^{-1} \text { let } \mathrm{A}=\left(\begin{array}{ccc}
a & b & c \\
d & e & f \\
g & h & i
\end{array}\right) \text {. Then } v \times v^{-1}=I \\
& \Rightarrow\left(\begin{array}{lll}
a & b & c \\
d & e & f \\
g & h & i
\end{array}\right) \times\left[\begin{array}{ccc}
\mathcal{W}_{11} & 0 & 0 \\
\mathcal{W}_{22} & \mathcal{W}_{33} & \mathcal{W}_{44} \\
0 & 0 & \lambda_{v}
\end{array}\right]=\left(\begin{array}{lll}
1 & 0 & 0 \\
0 & 1 & 0 \\
0 & 0 & 1
\end{array}\right) \\
& =\left(\begin{array}{lll}
a w 11+b w 22 & b w 33 & b w 44+c h v \\
d w 11+e w 22 & e w 33 & e w 44+f h v \\
g w 11+h w 22 & h w 33 & h w 44+i h v
\end{array}\right)=\left(\begin{array}{lll}
1 & 0 & 0 \\
0 & 1 & 0 \\
0 & 0 & 1
\end{array}\right) \\
& \Rightarrow\left\{\begin{array}{c}
a w 11+b w 22=1 \\
b w 33=0 \\
b w 44+c h v=0
\end{array} \Rightarrow b=c=0 \text { and } a=\frac{1}{w 11}\right. \\
& \left\{\begin{array}{c}
d w 11+e k 22=0 \\
e w 33=1 \\
e w 44+f h v=0
\end{array} \Rightarrow d=\frac{-w 22}{w 11 w 33}, f=\frac{-w 44}{w 33 h v} \text { and } e=\frac{1}{w 33}\right.
\end{aligned}
$$

$$
\left\{\begin{array}{c}
g w 11+h w 22=0 \\
h w 33=0 \\
h w 44+i h v=1
\end{array} \Rightarrow h=g=0 \text { and } i=\frac{1}{h v}\right.
$$$$
\Rightarrow v^{-1}=\left(\begin{array}{ccc}
\frac{1}{w 11} & 0 & 0 \\
\frac{-w 22}{w 11 w 33} & \frac{1}{w 33} & \frac{-w 44}{w 33 h v} \\
0 & 0 & \frac{1}{h v}
\end{array}\right)
$$

$$
\text { Hence } \begin{aligned}
F v^{-1}= & {\left[\begin{array}{ccc}
0 & 0 & a_{2} b m_{S_{h 2}} \\
0 & 0 & 0 \\
c a_{2} & 0 & 0
\end{array}\right] \times\left(\begin{array}{ccc}
\frac{1}{w 11} & 0 & 0 \\
\frac{-w 22}{w 11 w 33} & \frac{1}{w 33} & \frac{-w 44}{w 33 h v} \\
0 & 0 & \frac{1}{h v}
\end{array}\right) } \\
& =\left(\begin{array}{ccc}
0 & 0 & \frac{a_{2} b m}{h v} \\
0 & 0 & 0 \\
\frac{c a_{2}}{w 11} & 0 & 0
\end{array}\right)
\end{aligned}
$$

Then the spectral radius (the dominant Eigen value) $F v^{-1}$ is equal to $R_{0}$.

$$
\text { That is }|J-\lambda I|=0
$$




$$
\begin{gathered}
\Rightarrow\left|\begin{array}{ccc}
-\lambda & 0 & \frac{a_{2} b m}{\lambda_{v}} \\
0 & -\lambda & 0 \\
\frac{c a_{2}}{w_{11}} & 0 & -\lambda
\end{array}\right|=0 \\
\Rightarrow-\lambda^{3}+\frac{a_{2}^{2} b c m}{w_{11} \lambda_{v}} \lambda=0 \\
\quad=-\lambda\left(\lambda^{2}-\frac{a_{2}^{2} b c m}{w_{11} \lambda_{v}}\right)=0 \\
\Rightarrow \lambda=0 \text { or } \lambda^{2}=\frac{a_{2}^{2} b c m}{w_{11} \lambda_{v}}=\frac{a_{2}^{2} b c m}{\left(\delta_{2}+r_{2}-\varepsilon_{1}\right) \lambda v}
\end{gathered}
$$

Therefore the dominant Eigen values of $F v^{-1}$ is $\lambda^{2}=\frac{a_{2}^{2} b c m}{\left(\delta_{2}+r_{2}-\varepsilon_{1}\right) \lambda v}$

$$
\Rightarrow R_{01}^{2}=\frac{a_{2}^{2} b c m}{w 11 \lambda v} \text {, then } R_{01}=\sqrt{\frac{a_{2}^{2} b c m}{w 11 \lambda v}}(*)
$$

3.3.7. Local Stability analysis (ih2*, $\mathbf{s h}^{*}$, iv $\left.2 *\right)=(0,1,0)$

To determine the stability of the system, we will consider linearizing the systems of equations (3.5.14)- (3.5.17) about the equilibrium points by taking the Jacobian of them.

The Jacobian matrix at any point (ih2*,sh2*,iv2*) is given by

$$
\begin{aligned}
& J\left(i_{h 2}^{*}, S_{h 2}^{*}, i_{v}^{*}\right)= \\
& {\left[\begin{array}{ccc}
-\left(r_{2}+\delta_{2}-\varepsilon_{1}\right)+2 \delta_{2} i_{h 2} & a_{2} b m i_{v} & a_{2} b m S_{h 2} \\
-v_{2}+\delta_{2} S_{h 2} & -a_{2} b m i_{v}+\delta_{2} i_{h 2}-\varepsilon_{1}-v_{2} & -a_{2} b m S_{h 2} \\
a_{2} c\left(1-i_{v}\right) & 0 & -a_{2} c i_{h 2}-\lambda_{v}
\end{array}\right]}
\end{aligned}
$$

Then the Jacobian matrix of the above matrix at DFE point $(0,1,0)$ is given by

$$
\begin{aligned}
& J(0,1,0)=\left[\begin{array}{ccc}
-\left(r_{2}+\delta_{2}-\varepsilon_{1}\right) & 0 & a_{2} b m \\
-v_{2}+\delta_{2} & -\varepsilon_{1}-v_{2} & -a_{2} b m \\
a_{2} c & 0 & -\lambda_{v}
\end{array}\right] \text { (ii) } \\
& \text { Now applying }\left|J-\lambda I_{3}\right|=0 \\
& \Rightarrow\left[\begin{array}{ccc}
-\left(r_{2}+\delta_{2}-\varepsilon_{1}\right)-\lambda & 0 & a_{2} b m \\
-v_{2}+\delta_{2} & -\varepsilon_{1}-v_{2}-\lambda & -a_{2} b m \\
a_{2} c & 0 & -\lambda_{v}-\lambda
\end{array}\right] \text { (iii) }
\end{aligned}
$$

The corresponding characteristic equation of (iii) is

$$
\lambda^{3}+b_{1} \lambda^{2}+b_{2} \lambda+b_{3}=0
$$

Where $b 1, b 2$, and $b 3$ are the coefficients given by the expressions:

$$
\begin{gathered}
b_{3}=\delta_{2} \varepsilon_{1} \lambda_{v}+r_{2} v_{2} \lambda_{v}+\varepsilon_{1}^{2} \lambda_{v}+a_{2}^{2} b c m\left(v_{2}+\varepsilon 2\right)+r_{2} \varepsilon_{1} \lambda_{v}+\delta_{2} v_{2} \lambda_{v}+\varepsilon_{1} v_{2} \lambda_{v} \\
b_{2}=\left(\frac{v_{2}}{R_{01}^{2} \lambda_{v}}-1\right)+\left(\delta_{2}-\varepsilon_{1}+r_{2}\right) \varepsilon_{1}+\left(r_{2}+v_{2}\right) \lambda_{v} \\
b_{1}=v_{2}+r_{2}+\lambda_{v}+\delta_{2}
\end{gathered}
$$

Using Routh-Hurwitz criteria (Edelstein-Keshet 1988), the equilibrium is locally stable if the following conditions are satisfied. If these conditions are true, then all roots of the characteristic polynomial equation have negative real part, which concludes that there is a stable equilibrium. 
The conditions are:

(i) $b_{1}>0$

(ii) $b_{3}>0$ and

(iii) $b_{1} b_{2}-b_{3}>0$ must hold.

To show (i), (ii), and (iii) we use

(i) $b_{1}>0$

Clearly $b_{1}>0$ since all parameters are positive.

(ii) $b_{3}>0$

$$
\begin{aligned}
& b_{3}=\delta_{2} \varepsilon_{1} \lambda_{v}+r_{2} v_{2} \lambda_{v}-\varepsilon_{1}^{2} \lambda_{v}-a 2 b c m\left(v_{2}+\varepsilon_{1}\right)+r_{2} \varepsilon_{1} \lambda_{v}+\delta_{2} \varepsilon_{1} \lambda_{v}-\varepsilon_{1} v_{2} \lambda_{v} \\
& =\left(\delta_{2}+r_{2}-\varepsilon_{1}\right) v_{2} \lambda_{v}-a_{2}^{2} b c m_{v_{2}}-a_{2}^{2} b c m_{\varepsilon_{1}}+r_{2} \varepsilon_{1} \lambda_{v}+\delta_{2} \varepsilon_{1} \lambda_{v}-\varepsilon_{1}^{2} \lambda_{v} \\
& =\left(\left(\delta_{2}+r_{2}-\varepsilon_{1}\right) \lambda_{v}-a 2 b c m\right)_{2}-a_{2}^{2} b c m_{\varepsilon_{1}}+r_{2} \varepsilon_{1} \lambda_{v}+\delta_{2} \varepsilon_{1} \lambda_{v}-\varepsilon_{1}^{2} \lambda_{v} \\
& =\left(r_{2}+\delta_{2}-\varepsilon_{1}\right) \lambda_{v} v_{2}-a_{2}^{2} b c m v_{2}+\left(\delta_{2}+r_{2}-\varepsilon_{1}\right) \lambda_{v} \varepsilon_{1}-a_{2}^{2} b c m \varepsilon_{1} \\
& =\left(\frac{1}{R_{01}^{2}}-1\right) v_{2}+\left(\delta_{2}+r_{2}-\varepsilon_{1}\right) \lambda_{v} \varepsilon_{1}-a_{2}^{2} b c m \varepsilon_{1} \\
& =\left(\frac{1}{R_{01}^{2}}-1\right) v_{2}+\left(\frac{1}{R_{01}^{2}}-1\right) \varepsilon_{1} \\
& =\left(\frac{1}{R_{01}^{2}}-1\right)\left(\varepsilon_{1}+v_{2}\right)
\end{aligned}
$$

Clearly we see that the disease free equilibrium point is locally asymptotically stable if $R_{01}^{2}<1$. If $R_{01}^{2}>1$ then $b_{3}<0$. Hence, there is only one sign change in the sequence, $b_{1}, b_{2}, b_{3}$ and so by Descartes rule of sign, there is one eigenvalue with positive real part. This implies that the disease-free equilibrium point $(0,1,0)$ is unstable. (i.e Since the second condition failed then the DFE point unstable). Finally, we show that $b_{1} b_{2}-b_{3}>0$. The expression for $b_{2}$ is

$b_{2}=\left(\frac{v_{2}}{R_{01}^{2} \lambda_{v}}-1\right)+\left(\delta_{2}-\varepsilon_{1}+r_{2}\right) \varepsilon_{1}+\left(r_{2}+v_{2}\right) \lambda_{v}$

(iii) $b_{1} b_{2}-b_{3}>0$

$\left(\left(\frac{v_{2}}{R_{01}^{2} \lambda_{v}}-1\right)+\left(\delta_{2}-\varepsilon_{1}+r_{2}\right) \varepsilon_{1}+\left(r_{2}+v_{2}\right) \lambda_{v}\right)\left(r_{2}+v_{2}+\lambda_{v}+\delta_{2}\right)-\left(\frac{1}{R_{01}^{2}}-1\right)\left(\varepsilon_{1}+v_{2}\right)$

3.3.8. Calculating Reproductive Number $R_{02}$ for the third age group

$$
\begin{aligned}
& \frac{d i_{h 3}}{d t}=a_{3} b m_{S_{h 3} i_{v 2}}-\left(r_{3}+\delta_{3}+\varepsilon_{2}\right) i_{h 3}+\varepsilon_{2} i_{h 2}+\delta_{3} i_{h 3}^{2} \\
& \frac{d s_{h 3}}{d t}=-a_{3} b m S_{h 3} i_{v 3}-\varepsilon_{2} S_{h 3}+\varepsilon_{2} S_{h 2}+\varepsilon_{2} S_{h 2}+v_{3}\left(1-S_{h 3}-i_{h 3}\right)+\delta_{3} S_{h 3} i_{h 3} \\
& \frac{d i_{v 1}}{d t}=c a_{3}\left(1-i_{v 3}\right) i_{h 3}-\lambda_{v} i_{v 3}
\end{aligned}
$$

To determine the reproduction number it is important to distinguish new infections from all other changes in population.Forming the matrices for $\mathrm{F}$ and $\mathrm{V}$ at the disease free equilibrium of the form $\mathrm{E} 0=\left(\mathrm{ih} 3 *, \mathrm{sh}^{*}, \mathrm{iv} *\right)=(0,1,0$,$) such that there is no infection in the population,$ 


\section{Gains terms}

$F_{1}=a_{3} b m s_{h 3} i_{v 3} F_{2}=0 F_{3}=c a_{3}\left(1-i_{v 3}\right) i_{h 3}$

\section{Loss terms}

$$
\begin{aligned}
& v_{1}=\left(r_{3}+\delta_{3}+\varepsilon_{2}\right) i_{h 3}+\delta_{1} i_{h 2}^{2}+\varepsilon_{2} i_{h 2}+\delta_{3} i_{h 3}^{2} \\
& v_{2}=a_{3} b m_{S_{h 3}} i_{v 3}+v_{3}\left(1-S_{h 3}-i_{h 3}\right)+\delta_{3} S_{h 3} i_{h 3}+\varepsilon_{2} S_{h 3}+\varepsilon_{2} S_{h 2} \\
& v_{3}=\lambda_{v} i_{v 3}
\end{aligned}
$$

Then the Jacobian matrix of $F$ (gain terms) is given by

$$
F\left(i_{h 3}, s_{h 3}, i_{v 3}\right)=\left[\begin{array}{ccc}
0 & a_{3} b m i_{v 3} & a_{3} b m s_{h 3} \\
0 & 0 & 0 \\
c a_{3}\left(1-i_{v 3}\right) & 0 & -a_{3} c i_{h 3}
\end{array}\right]
$$

And the Jacobian matrix of $\mathrm{F}$ (loss terms) is given by

$$
\begin{gathered}
v\left(i_{h 3}{ }^{*}, s_{h 3} * i_{v} *\right)=\left[\begin{array}{ccc}
m_{1} & 0 & 0 \\
m_{2} & m_{3} & m_{4} \\
0 & 0 & \lambda_{v}
\end{array}\right] \\
\text { Where } \\
m_{1}=\delta_{3}+r_{3}+\varepsilon_{2}+2 \delta_{3} i_{h 3} \\
m_{12}=v_{3}+\delta_{3} S_{h 3} \\
m_{3}=\varepsilon_{2}+v_{3}+a_{3} b m i_{v}+\delta_{3} i_{h 3} \\
m_{4}=a_{3} b m s_{h 3}
\end{gathered}
$$

Then the Jacobian matrix at DFE point of $(0,1,0)$ is given by

$$
\begin{aligned}
& V(0,1,0)=\left[\begin{array}{ccc}
m_{11} & 0 & 0 \\
m_{12} & m_{13} & m_{14} \\
0 & 0 & \lambda_{v}
\end{array}\right] \\
& \text { Where } \\
& m_{11}=\delta_{3}+r_{3}+\varepsilon_{2} \\
& m_{12}=v_{3}+\delta_{3} \\
& m_{13}=\varepsilon_{2}+v_{3} \\
& m_{14}=a_{3} b m \\
& \text { Now to find } v^{-1} \text { let } v^{-1}=\left(\begin{array}{ccc}
a & b & c \\
d & e & f \\
g & h & i
\end{array}\right) \text {. Then } v \times v^{-1}=I_{3} \\
& \Rightarrow\left(\begin{array}{lll}
a & b & c \\
d & e & f \\
g & h & i
\end{array}\right) \times\left[\begin{array}{ccc}
m_{11} & 0 & 0 \\
m_{12} & m_{13} & m_{14} \\
0 & 0 & \lambda_{v}
\end{array}\right]=\left(\begin{array}{lll}
1 & 0 & 0 \\
0 & 1 & 0 \\
0 & 0 & 1
\end{array}\right) \\
& \Rightarrow\left(\begin{array}{lll}
a m_{11}+b m_{22} & b m_{33} & b m_{44}+c \lambda_{v} \\
d m_{11}+e m_{22} & e m_{33} & e m_{44}+f \lambda_{v} \\
g m_{11}+h m_{22} & h m_{33} & h m_{44}+i \lambda_{v}
\end{array}\right)=\left(\begin{array}{lll}
1 & 0 & 0 \\
0 & 1 & 0 \\
0 & 0 & 1
\end{array}\right)
\end{aligned}
$$




$$
\begin{aligned}
& \left\{\begin{array}{c}
a m_{11}+b m_{22}=1 \\
b m_{33}=0 \\
b m_{44}+c \lambda_{v}=0
\end{array} \Rightarrow b=c=0 \text { and } a=\frac{1}{m_{11}}\right. \\
& \left\{\begin{array}{c}
d m_{11}+e m_{22}=0 \\
e m_{33}=1 \\
e m_{44}+f \lambda_{v}=0
\end{array} \Rightarrow d=\frac{-m_{22}}{m_{11} m_{22}} \quad f=\frac{-m_{44}}{m_{33} \lambda_{v}} \text { and } e=\frac{1}{m_{33}}\right. \\
& \left\{\begin{array}{c}
g m_{11}+h m_{22}=0 \\
h m_{33}=0 \\
h m_{44}+i \lambda_{v}=1
\end{array} \Rightarrow h=g=0 \text { and } i=\frac{1}{\lambda_{v}}\right. \\
& \Rightarrow v^{-1}=\left(\begin{array}{ccc}
\frac{1}{m_{11}} & 0 & 0 \\
\frac{-w_{22}}{m_{11} m_{33}} & \frac{1}{m_{33}} & \frac{-m_{44}}{m_{33} \lambda_{v}} \\
0 & 0 & \frac{1}{\lambda_{v}}
\end{array}\right) \\
& \text { Hence } F v^{-1}=\left[\begin{array}{ccc}
0 & 0 & a_{3} b m s_{h 3} \\
0 & 0 & 0 \\
c a_{3} & 0 & 0
\end{array}\right] \times\left(\begin{array}{ccc}
\frac{1}{m_{11}} & 0 & 0 \\
\frac{-w_{22}}{m_{11} m_{33}} & \frac{1}{m_{33}} & \frac{-m_{44}}{m_{33} \lambda_{v}} \\
0 & 0 & \frac{1}{\lambda_{v}}
\end{array}\right) \\
& =\left(\begin{array}{ccc}
0 & 0 & \frac{a_{3} b m}{\lambda_{v}} \\
0 & 0 & 0 \\
\frac{c a_{3}}{m_{11}} & 0 & 0
\end{array}\right)
\end{aligned}
$$

Then the spectral radius (the dominant eigen value) $F v^{-1}$ is equal to $R_{0}$.

Obtaining the eigenvalues as usual form of the formula $|J-\lambda I|=0$

$$
\begin{aligned}
& \Rightarrow\left|\begin{array}{ccc}
-\lambda & 0 & \frac{a_{3} b m}{\lambda_{v}} \\
0 & -\lambda & 0 \\
\frac{c a_{3}}{m_{11}} & 0 & -\lambda
\end{array}\right|=0 \\
& \Rightarrow-\lambda^{3}+\frac{a_{3}^{2} b c m}{m_{11} \lambda_{v}} \lambda=0 \\
& =-\lambda\left(\lambda^{2}-\frac{a_{3}^{2} b c m}{m_{11} \lambda_{v}}\right)=0
\end{aligned}
$$




$$
\Rightarrow \lambda=0 \text { or } \lambda^{2}=\frac{a_{3}^{2} b c m}{m_{11} \lambda_{v}} .
$$

Therefore the dominant Eigen values of $F v^{-1}$ is $\lambda^{2}=\frac{a_{3}^{2} b c m}{m_{11} \lambda_{v}}$

$$
\Rightarrow R_{02}^{2}=\frac{a_{3}^{2} b c m}{m_{11} \lambda_{v}}, \text { then } R_{02}=\sqrt{\frac{a_{3}^{2} b c m}{m_{11} \lambda_{v}}}
$$

Clearly if $a_{3}^{2} b c m>m_{11} \lambda_{v}$, then the reproduction number is $R_{02}^{2}>1$ and the disease is highly affect the community. If $a_{3}^{2} b c m<m_{11} \lambda_{v}$ then the reproduction number is $R_{02}^{2}<1$ and the malaria disease is die out. In the context of epidemiology modeling, it is generally known that if $R_{0}<1$, then the disease free equilibrium is locally asymptotically stable and the disease will be eradicated from the community.

\subsection{Local Stability analysis of DFE point (ih3*,sh3*,iv*) $=(0,1,0)$}

To determine the stability of the system, we will consider linearizing the systems of equations (3.5.18)- (3.5.20) about the equilibrium points by taking the Jacobian of them.

The Jacobian matrix at any point (ih3*,sh3*,iv3*) is given by

$$
\begin{aligned}
& J\left(i_{h 3}{ }^{*}, S_{h 3}{ }^{*}, i_{v}{ }^{*}\right)= \\
& {\left[\begin{array}{ccc}
-\left(r_{3}+\delta_{3}+\varepsilon_{2}\right)+2 \delta_{3} i_{h 3} & a_{3} b m i_{v} & a_{3} b m s_{h 3} \\
-v_{3}+\delta_{3} S_{h 3} & -a_{3} b m i_{v}+\delta_{3} i_{h 3}-v_{3}-\varepsilon_{2} & -a_{3} b m s_{h 3} \\
c a_{3}\left(1-i_{v}\right) & 0 & -c a_{3} i_{h 3}-\lambda_{v}
\end{array}\right]}
\end{aligned}
$$

Then the Jacobian matrix of the above matrix at DFE point $(0,1,0)$ is given by

$$
J(0,1,0)=\left[\begin{array}{ccc}
-\left(r_{3}+\delta_{3}+\varepsilon_{2}\right) & 0 & a_{3} b m \\
-v_{3}+\delta_{3} & -\left(v_{3}+\varepsilon_{2}\right) & -a_{3} b m \\
c a_{3} & 0 & -\lambda_{v}
\end{array}\right] \text { (ii) }
$$

The corresponding characteristic polynomial is

$$
\left|\begin{array}{ccc}
-\left(r_{3}+\delta_{3}+\varepsilon_{2}\right)-\lambda & 0 & a_{3} b m \\
-v_{3}+\delta_{3} & -\left(v_{3}+\varepsilon_{2}\right)-\lambda & -a_{3} b m \\
c a_{3} & 0 & -\lambda_{v}-\lambda
\end{array}\right|=0 \text { (iii) }
$$

The corresponding characteristic equation (iii) is given by

$$
\lambda^{3}+d_{1} \lambda^{2}+d_{2} \lambda+d_{3}=0
$$

Where $d 1, d 2$ and $d 3$ are the coefficients given by the expressions:

$$
\begin{gathered}
d_{3}=-a_{3}^{2} b c m \varepsilon_{2}-a_{3}^{2} b c m v_{3}+\delta_{3} v_{3} \lambda_{v}+\varepsilon_{2}^{2} \lambda_{v}+r_{3} v_{3} \lambda_{v}+r_{3} \varepsilon_{2} \lambda_{v}+\delta_{3} \varepsilon_{2} \lambda_{v}+v_{3} \varepsilon_{2} \lambda_{v} \\
d_{2}=\left(\frac{1}{R_{02}^{2}}-1\right)+\varepsilon_{2} v_{3}+\varepsilon_{2}^{2}+r_{3} \varepsilon_{2}+r_{3} v_{3}+\lambda_{v} v_{3}+\delta_{3} \varepsilon_{2}+\lambda_{v} \varepsilon_{2}+\delta_{3} v_{3} \\
d_{1}=v_{3}+\lambda_{v}+\delta_{3}+r_{3}+2 \varepsilon_{2}
\end{gathered}
$$

Using Routh-Hurwitz criteria (Edelstein-Keshet 1988), the equilibrium is locally stable if the following conditions are satisfied. If these conditions are true, then all roots of the characteristic polynomial equation have negative real part, which concludes that there is a stable equilibrium.

The three conditions are:

$$
\begin{array}{ll}
\text { (i) } & d_{1}>0 \\
\text { (ii) } & d_{3}>0
\end{array}
$$

(iii) $d_{1} d_{2}-d_{3}>0$ must hold.

To show (i), (ii), and (iii) we use 


$$
\text { (i) } d_{1}=v_{3}+\lambda_{v}+\delta_{3}+r_{3}+2 \varepsilon_{2}
$$

Clearly $d_{1}>0$ since all parameters have positive values. Now to check the second condition we check for

$$
\begin{gathered}
d_{3}>0 \\
d_{3}=-a_{3}^{2} b c m \varepsilon_{2}-a_{3}^{2} b c m v_{3}+\delta_{3} v_{3} \lambda_{v}+\varepsilon_{2}^{2} \lambda_{v}+r_{3} v_{3} \lambda_{v}+r_{3} \varepsilon_{2} \lambda_{v}+\delta_{3} \varepsilon_{2} \lambda_{v}+v_{3} \varepsilon_{2} \lambda_{v} \\
=\left(\left(\varepsilon_{2}+r_{3}+\delta_{3}\right) \lambda_{v}-a_{3}^{2} b c m\right)_{3}+\varepsilon_{2}^{2} \lambda_{v}+r_{3} \varepsilon_{2} \lambda_{v}+\delta_{3} \varepsilon_{2} \lambda_{v}-a_{3}^{2} b c m \mathcal{E}_{2} \\
=\left(\frac{1}{R_{02}^{2}}-1\right) v_{3}+\varepsilon_{2}^{2} \lambda_{v}+r_{3} \varepsilon_{2} \lambda_{v}+\delta_{3} \varepsilon_{2} \lambda_{v}-a_{3}^{2} b c m \varepsilon_{2} \\
=\left(\frac{1}{R_{02}^{2}}-1\right) v_{3}+\left(\varepsilon_{2}+r_{3}+\delta_{3}\right) \lambda_{v} \varepsilon_{2}-a_{3}^{2} b c m \varepsilon_{2} \\
=\left(\frac{1}{R_{02}^{2}}-1\right) v_{3}+\left(\left(\varepsilon_{2}+r_{3}+\delta_{3}\right) \lambda_{v}-a_{3}^{2} b c m\right) \varepsilon_{2} \\
=\left(\frac{1}{R_{02}^{2}}-1\right) v_{3}+\left(\frac{1}{R_{02}^{2}}-1\right) \varepsilon_{2} \\
\left.=\left(\frac{1}{R_{02}^{2}}-1\right) v_{3}+\varepsilon_{2}\right)
\end{gathered}
$$

So we have seen that the disease free equilibrium point is locally asymptotically stable if $R_{02}<1$. If $R_{02}^{2}>1$, we can see that $d_{3}<0$. Hence, there is only one sign change in the sequence, $d_{1}, d_{2}, d_{3}$ and so by Descartes rule of sign, there is one eigenvalue with positive real part. This implies that the disease-free equilibrium point $(0,1,0)$ is unstable. (i.e Since the second condition failed then the DFE point unstable)

Finally, we show that $d_{1} d_{2}-d_{3}>0$. The expression for $d 2$ is

$d_{2}=\left(\frac{1}{R_{02}^{2}}-1\right)+\varepsilon_{2} v_{3}+\varepsilon_{2}^{2}+r_{3} \varepsilon_{2}+r_{3} v_{3}+\lambda_{v} v_{3}+\delta_{3} \varepsilon_{2}+\lambda_{v} \varepsilon_{2}+\delta_{3} v_{3}$

Hence we get

$d_{1} d_{2}-d_{3}=$

$\left[\quad v_{3}+\lambda_{v}+\delta_{3}+r_{3}+2 \varepsilon_{2} \quad\right]^{*}\left[\left(\frac{1}{R_{02}^{2}}-1\right)+\varepsilon_{2} v_{3}+\varepsilon_{2}^{2}+r_{3} \varepsilon_{2}+r_{3} v_{3}+\lambda_{v} v_{3}+\delta_{3} \varepsilon_{2}+\lambda_{v} \varepsilon_{2}+\delta_{3} v_{3} \quad\right] *$ $\left.-\left(\frac{1}{R_{02}^{2}}-1\right) v_{3}+\varepsilon_{2}\right)$

Thus the third condition $d_{1} d_{2}-d_{3}>0$ also satisfied if and only if $R_{02}^{2}<1$. If $R_{02}^{2}>1$, then $d_{1} d_{2}-d_{3}<0$.

\subsubsection{Local Stability analysis of endemic equilibrium points for the age group three}

The endemic equilibrium state is the state where the disease cannot be totally eradicated but remains in the population.

For the disease to persist in the population, the susceptible class, the Infectious class and the Recovered Class must not be zero at equilibrium state (i.e $i_{h 1}, i_{v} \neq 0$ ).

In other words, if $E_{1}$ is the endemic equilibrium state, then in (i.e. equilibrium where the infected components of the age-structured model (3.5.18) - (3.5.20) are non-zero or where the disease persist in the population (all state variables are positive) will be derived. When the disease free equilibrium point is unstable, then there exists an endemic equilibrium point

The endemic equilibrium solution of (3.5.18)-(3.5.20) can be found by setting the right hand side to zero and solving the nonlinear system to obtain 


$$
\begin{gathered}
\frac{d i_{h 3}}{d t}=a_{3} b m_{S_{h 3}} i_{v 2}-\left(r_{3}+\delta_{3}+\varepsilon_{2}\right) i_{h 3}+\varepsilon_{2} i_{h 2}+\delta_{3} i_{h 3}^{2}(*) \\
\frac{d S_{h 3}}{d t}=-a_{3} b m s_{h 3} i_{v 3}-\varepsilon_{2} S_{h 3}+\varepsilon_{2} S_{h 2}+\varepsilon_{2} S_{h 2}+v_{3}\left(1-s_{h 3}-i_{h 3}\right)+\delta_{3} S_{h 3} i_{h 3}(* *) \\
\frac{d i_{v 1}}{d t}=c a_{3}\left(1-i_{v 3}\right) i_{h 3}-\lambda_{v} i_{v 3}(* * *) \\
\text { Now from (***) we have that } i_{v}=\frac{a_{3} c i_{h 3}}{a_{3} c i_{h 3}+\lambda_{v}} \text { (i) }
\end{gathered}
$$

Substitute (i) in $(*)$ and $(* *)$ solve for ih $3 *$.

Then the Jacobian matrix of age group one model at endemic steady state $\left(i_{h 3} *, s_{h 3} *, i_{v} *\right)$ given by:

$$
\begin{aligned}
& J\left(i_{h 3}{ }^{*}, s_{h 3} *, i_{v} *\right)= \\
& {\left[\begin{array}{ccc}
-\left(r_{3}+\delta_{3}+\varepsilon_{2}\right)+2 \delta_{3} i_{h 3} & a_{3} b m i_{v} & a_{3} b m s_{h 3} \\
-v_{3}+\delta_{3} S_{h 3} & -a_{3} b m i_{v}+\delta_{3} i_{h 3}-v_{3}-\varepsilon_{2} & -a_{3} b m s_{h 3} \\
c a_{3}\left(1-i_{v}\right) & 0 & -c a_{3} i_{h 3}-\lambda_{v}
\end{array}\right]}
\end{aligned}
$$

Then see the EEP of stability for this model in chapter four.

\section{NUMERICAL SIMULATION}

We present numerical simulations using Matlab to illustrate the transmission of malaria disease for the three age group described above. The parameter values are given in Table 2.

\subsection{Numerical simulation of the first age group model}

Consider the model given by equation (3.5.12)-(3.5.14)

$$
\begin{gathered}
\frac{d i_{h 1}}{d t}=a b m s_{h 1} i_{v}-\left(r_{1}+\delta_{1}+\lambda_{h}\right) i_{h 1}+\delta_{1} i_{h 1}^{2}(\mathrm{a}) \\
\frac{d s_{h 1}}{d t}=\lambda_{h}\left(1-s_{h 1}\right)-a b m s_{h 1} i_{v}+v_{1}\left(1-s_{h 1}-i_{h 1}\right)+\delta_{1} s_{h 1} i_{h 1}(\mathrm{~b}) \\
\frac{d i_{v}}{d t}=c a_{1}\left(1-i_{v}\right) i_{h 1}-\lambda_{v} i_{v}(\mathrm{c})
\end{gathered}
$$

\subsection{Reproduction number of age group one}

The reproduction number measures the average number of new infected population by a single infected population. Since we have shown an explicit expression for $R 0$, we can analytically evaluate its sensitivity to the different parameter values.

We can also numerically evaluate the sensitivity of the endemic equilibrium to the parameter values, as this will allow us to determine the relative importance to the age-structured malaria transmission and prevalence.

$$
\begin{gathered}
R_{0}^{2}=\frac{a_{1}^{2} b c m}{k 11 h v} \text { or } R_{0}=\sqrt{\frac{a_{1}^{2} b m c}{k 11 h v}} \\
\Rightarrow R_{0}=\sqrt{\frac{(0.46)^{2}(0.5)(0.48)(0.2)}{(0.071)(0.00012+0.0014+0.00000214)}}=9.6944>1
\end{gathered}
$$

Since $R_{0}>1$, the prevalence of malaria disease will result in an epidemic. This is due to the fact that the rate of transmission is greater than the recovery rate. 


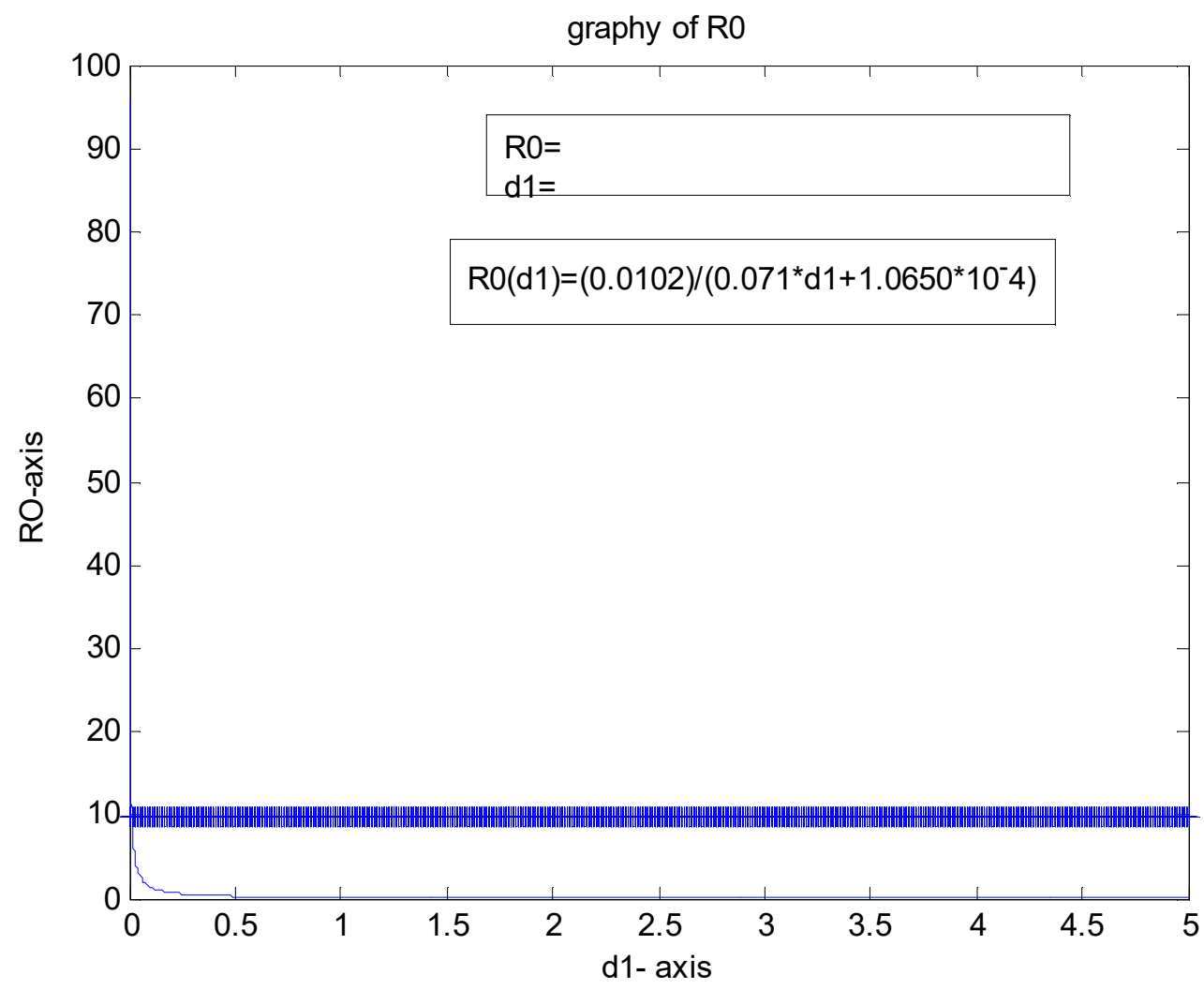

Figure 5.1 graph of the basic reproduction number $R_{0}$

From this graph we can observe that if the Per capita death rate $\delta_{1}$ increases and $\lambda_{h}, r_{1}$ is constant then the reproduction number $R_{0}$ decreases. This means that when the Per capita death rate $\delta_{1}$ decrease then the reproduction number is increasing and the susceptible population increasing. And when the Per capita death rate $\delta_{1}$ increases $R_{0}$ decreases that means when the when the Per capita death rate increase then the number of infected population decrease since individual in the infected class dies out. Similarly if $\lambda_{h}, \delta_{1}$ are constant and $r_{1}$ increases, then the reproduction number $R_{0}$ decreases and as $r_{1}$ decreases $R_{0}$ increases.

\subsection{Stability analysis of DFE point of first age group}

The Jacobian matrix of the given model at DFE point $(0$, sh 1,0$)$ is given as follows

$$
J(0, s h 1,0)=\left[\begin{array}{ccc}
-\left(\delta_{1}+r_{1}+\lambda_{h}\right) & 0 & a_{1} b m_{S_{h 1}} \\
-v_{1}+\delta_{1} S_{h 1} & 0 & -a_{1} b m_{S_{h 1}} \\
c a_{1} & 0 & -\lambda_{v}
\end{array}\right]
$$

And the Jacobian matrix at DFE point $(0,1,0)$ is

$$
J(0,1,0)=\left[\begin{array}{ccc}
-0.0015 & 0 & 0.0460 \\
-0.0083 & -0.0084 & -0.0460 \\
-0.2208 & 0 & -0.071
\end{array}\right]
$$

The corresponding characteristic polynomial is $\left|J-\lambda I_{3}\right|=0$

$$
\Rightarrow\left|\begin{array}{ccc}
-0.0015-\lambda & 0 & 0.0460 \\
-0.0083 & -0.0084-\lambda & -0.0460 \\
-0.2208 & 0 & -0.071-\lambda
\end{array}\right|=0\left(^{*}\right)
$$


The characteristic equation of $(*)$ after calculation is given below

\begin{tabular}{|c|c|c|c|}
\multicolumn{5}{|c}{$\Rightarrow \lambda^{3}+0.0809 \lambda^{2}+0.0103 \lambda+0.0013=0$} \\
\hline$\lambda^{3}$ & 1 & 0.0103 & 0 \\
\hline$\lambda^{2}$ & 0.0809 & 0.0013 & 0 \\
\hline$\lambda^{1}$ & $b_{1}$ & $b_{2}$ & 0 \\
\hline$\lambda^{0}$ & $k_{1}$ & $k_{2}$ & 0 \\
\hline
\end{tabular}

And we can calculate out all the coefficients:

$$
\begin{gathered}
b_{1}=\frac{-1}{0.0809}\left|\begin{array}{cc}
1 & 0.0103 \\
0.0809 & 0.0013
\end{array}\right|=-0.0058 \\
b_{2}=\frac{-1}{0.0809}\left|\begin{array}{cc}
1 & 0 \\
0.0809 & 0
\end{array}\right|=0 \\
k_{1}=\frac{-1}{-0.0058}\left|\begin{array}{cc}
0.0809 & 0.0013 \\
-0.0058 & 0
\end{array}\right|=0.0013 \\
k_{2}=\frac{-1}{-0.0058}\left|\begin{array}{cc}
0.0809 & 0 \\
-0.0058 & 0
\end{array}\right|=0
\end{gathered}
$$

And filling these values into our Routh Array, we can determine whether the system is stable or not:

\begin{tabular}{|c|c|c|c|}
\hline$\lambda^{3}$ & 1 & 0.0103 & 0 \\
\hline$\lambda^{2}$ & 0.0809 & 0.0013 & 0 \\
\hline$\lambda^{1}$ & -0.0058 & 0 & 0 \\
\hline$\lambda^{0}$ & 0.0013 & 0 & 0 \\
\hline
\end{tabular}

Since the first columns do not contain the same sign, or the change of sign in the first column then by RouthHurwitz stability criteria the DFE point $(0,1,0)$ is unstable. Using Matlab we obtain the values of the Eigen value as follows.

The roots of the equation is

$$
\begin{gathered}
\lambda_{1}=0.0111+0.1117 i \\
\lambda_{2}=0.0111-0.1117 i \\
\lambda_{3}=-0.1032
\end{gathered}
$$

\subsection{Local Stability analysis of EE point of first age group}

The endemic equilibrium solution of (3.5.12)-(3.5.14) can be found by setting the right hand side to zero and solving the nonlinear system to obtain

$$
\begin{aligned}
& a b m s_{h 1} i_{v}-\left(r_{1}+\delta_{1}+\lambda_{h}\right) i_{h 1}+\delta_{1} i_{h 1}^{2}=0 \text { (a) } \\
& \lambda_{h}\left(1-s_{h 1}\right)-a b m s_{h 1} i_{v}+v_{1}\left(1-s_{h 1}-i_{h 1}\right)+\delta_{1} s_{h 1} i_{h 1}=0(\mathrm{~b}) \\
& c a_{1}\left(1-i_{v}\right) i_{h 1}-\lambda_{v} i_{v}=0 \text { (c) }
\end{aligned}
$$

Now from (c) we have that $i_{v}=\frac{a_{1} c i_{h 1}}{a_{1} c i_{h 1}+\lambda_{v}}$ (d)Substitute (d) in (a) and (b) solve for ih $1 *$ using Matlab we obtain $i h 1^{*}=0.3433$

Therefore the endemic equilibrium points for the respective states are: 
$\left(i_{h 1}^{*}, S_{h 1}^{*}, i_{v}^{*}\right)=(0.3433,0.0458,0.5164)$

The Jacobian matrix of (3.5.2)-(3.5.14) at any endemic steady state $\left(i_{h 1} *, s_{h 1}^{*}, i_{v}^{*}\right)$ is given by:

$J\left(i_{h 1}{ }^{*}, s_{h 1} * i_{v} *\right)=$

$$
\left[\begin{array}{ccc}
-\left(r_{1}+\delta_{1}+\lambda_{h}\right)+2 \delta_{1} i_{h 1} & -a_{1} b m i_{v} & a_{1} b m s_{h 1} \\
-v_{1}+\delta_{1} s_{h 1} & -\left(\lambda_{h}+v_{1}\right)-a_{1} b m i_{v}+\delta_{1} i_{h 1} & -a_{1} b m s_{h 1} \\
c a_{1}\left(1-i_{v}\right) & 0 & -c a_{1} i_{h 1}-\lambda_{v}
\end{array}\right]
$$

The Jacobian matrix of this equilibrium point is evaluated at $(0.9698,0.0107,0.7510)$ to obtain:

$$
J(0.9698,0.0107,0.7510)=\left[\begin{array}{ccc}
-0.0015 & -0.0238 & 0.0021 \\
-0.0083 & -0.0322 & -0.0021 \\
0.1068 & 0 & -0.1468
\end{array}\right]
$$

The characteristic polynomial is given by

$$
\left|\begin{array}{ccc}
-0.0015-\lambda & -0.0238 & 0.0021 \\
-0.0083 & -0.0322-\lambda & -0.0021 \\
0.1068 & 0 & -0.1468-\lambda
\end{array}\right|=0
$$

Solving for the roots of the polynomial in the Jacobian matrix leads to the characteristic equation:

$\lambda^{3}+0.1805 \lambda^{2}+0.0050 \lambda-1.6439 \times 10^{-5}=0$

Now constructing Routh-Hurwitz array to determine stability in the following ways

\begin{tabular}{|l|l|l|l|}
\hline$\lambda^{3}$ & \multicolumn{1}{|c|}{1} & 0.0050 & 0 \\
\hline$\lambda^{2}$ & 0.1805 & $-1.6439 \times 10^{-5}$ & 0 \\
\hline$\lambda^{1}$ & $b_{1}$ & $b_{2}$ & 0 \\
\hline$\lambda^{0}$ & $k_{1}$ & $k_{2}$ & 0 \\
\hline
\end{tabular}

And we can calculate out all the coefficients:

$$
\begin{aligned}
& b_{1}=\frac{-1}{0.1805}\left|\begin{array}{cc}
1 & 0.0050 \\
0.1805 & -1.6439 \times 10^{-5}
\end{array}\right|=0.0051 \\
& b_{2}=\frac{-1}{0.1805}\left|\begin{array}{cc}
1 & 0 \\
0.1805 & 0
\end{array}\right|=0 \\
& k_{1}=\frac{-1}{0.0051}\left|\begin{array}{ll}
0.1805 & -1.6439 \times 10^{-5} \\
0.0051 & 0
\end{array}\right|=-1.6439 \times 10^{-5} \\
& k_{2}=\frac{-1}{0.0051}\left|\begin{array}{ll}
0.1805 & 0 \\
0.0051 & 0
\end{array}\right|=0
\end{aligned}
$$

And filling these values into our Routh Array, we can determine whether the system is stable or not:

\begin{tabular}{|l|l|l|l|}
\hline$\lambda^{3}$ & \multicolumn{1}{|c|}{1} & 0.0050 & 0 \\
\hline$\lambda^{2}$ & 0.1805 & $-1.6439 \times 10^{-5}$ & 0 \\
\hline$\lambda^{1}$ & $\mathrm{~b} 1=0.0051$ & $\mathrm{~b} 2=0$ & 0 \\
\hline$\lambda^{0}$ & $\begin{array}{l}\mathrm{k} 1= \\
-1.6439 \times 10^{-5}\end{array}$ & $\mathrm{k} 2=0$ & 0 \\
\hline
\end{tabular}

Since all the first columns element contain the same sign, then by Routh-Hurwitz stability criteria the endemic equilibrium point is stable. Using Matlab we obtain the values of the Eigen value as follows.

The roots of the equation is 
$>>\mathrm{a}=\left[10.18050 .0050-1.6439 \times 10^{-5}\right]$;

$>\mathrm{f}=$ roots (a) $\lambda_{1}=-0.1453, \lambda_{2}=-0.0382, \lambda_{3}=0.0030$

All the roots dos not contain the same sign. Thus the EEP $(0.3433,0.0458,0.5164)$ is unstable by Routh-Hurwitz stability criteria.

4.2 Numerical simulation of the second age group model

Consider the model given by equation (3.5.15)-(3.5.17)

$$
\begin{aligned}
& \frac{d i_{h 2}}{d t}=a_{2} b m_{S_{h 2}} i_{v}-\left(r_{2}+\delta_{2}-\varepsilon_{1}\right) i_{h 2}-\varepsilon_{1} i h_{1}+\delta_{2} i_{h 2}^{2} \\
& \frac{d S_{h 2}}{d t}=-a_{2}{ }^{b m} S_{h 2} i_{v 2}-\varepsilon_{1} S_{h 1}+\delta_{2} S_{h 2} i_{h 2}-\varepsilon_{2} S_{h 2}+v_{2}\left(1-S_{h 2}-i_{h 2}\right)(\mathrm{ii}) \\
& \frac{d i_{v 2}}{d t}=c a_{2}\left(1-i_{v 2}\right) i_{h 2}-\lambda_{v} i_{v 2} \text { (iii) }
\end{aligned}
$$

\subsection{The reproduction number of age group two(5-25 years old)}

We have that $R_{01}{ }^{2}=\frac{a_{2}^{2} b c m}{w 11 \lambda v}$, or $R_{01}=\sqrt{\frac{a_{2}^{2} b m c}{w 11 \lambda v}}$

$$
\Rightarrow R_{01}=\sqrt{\frac{(0.4)^{2}(0.5)(0.48)(0.2)}{(0.071)(0.0035+0.0000000978-0.0000502)}}=5.5995>1
$$

Still the malaria transmission is present highly at this age level but less transmission than the first age group.

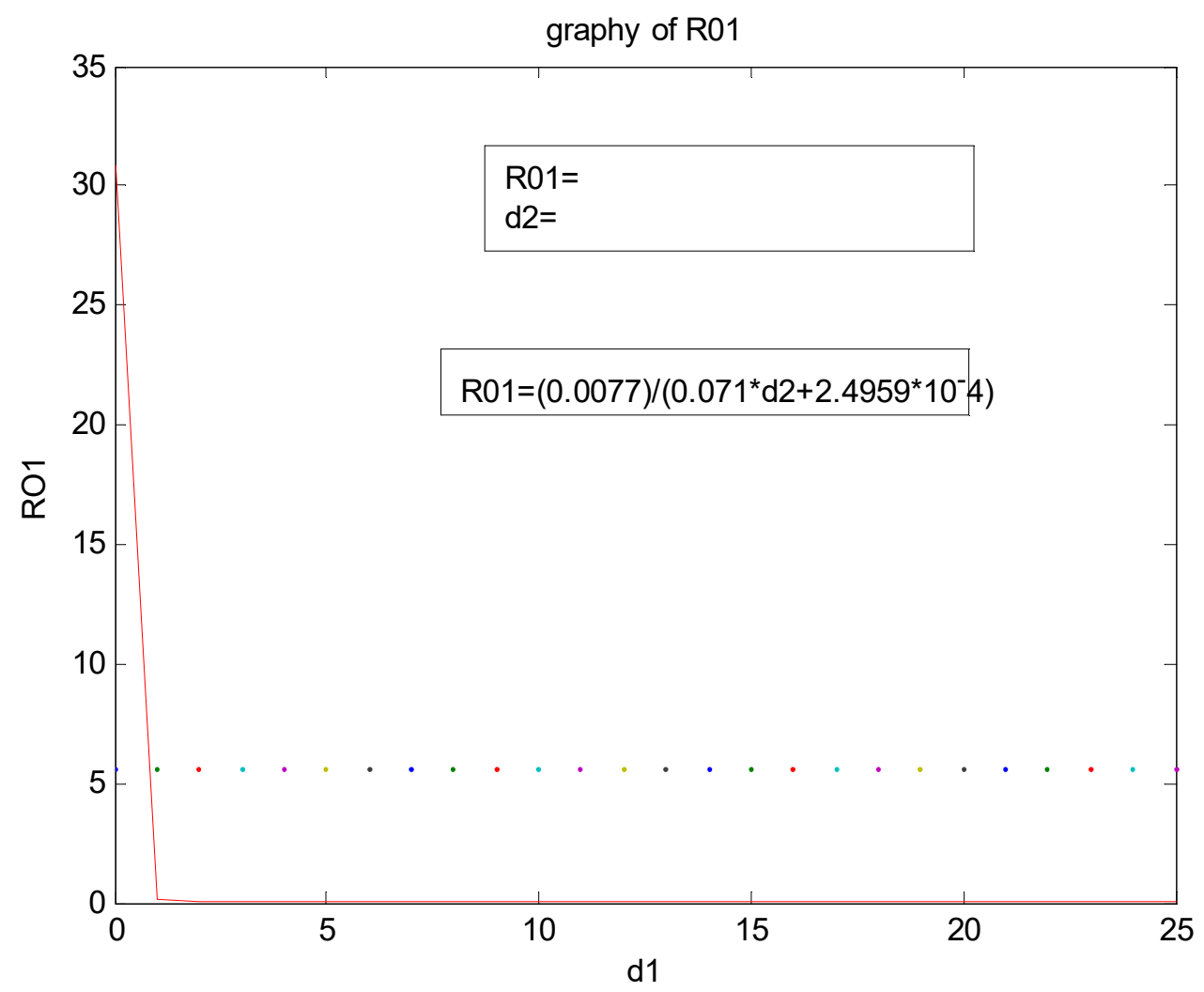

Figure 5.2 graph of the basic reproduction number $R_{01}$

From the above graph we can observe that if the Per capita death rate $\delta_{2}$ increases then the reproduction number $R_{01}$ decreases. This means that when the Per capita death rate $\delta_{2}$ decrease then the reproduction number is 
increasing and the susceptible population increasing. And when the Per capita death rate $\delta_{2}$ increases $R_{01}$ decreases that means when the when the Per capita death rate increase then the number of infected population decrease since individual in the infected class dies out.

\subsection{Local Stability analysis of DFE point of second age group}

Then the Jacobian matrix of the above matrix at DFE point $(0,1,0)$ is given by

$$
J(0,1,0)=\left[\begin{array}{ccc}
-\left(r_{2}+\delta_{2}-\varepsilon_{1}\right) & 0 & a_{2} b m \\
-v_{2}+\delta_{2} & -\left(v_{2}+\varepsilon_{1}\right) & -a_{2} b m \\
c a_{2} & 0 & -\lambda_{v}
\end{array}\right] \text { (ii) }
$$

The characteristic polynomial is given by $\left|J-\lambda I_{3}\right|=0$

$$
\begin{aligned}
& \Rightarrow\left|\begin{array}{ccc}
-\left(r_{2}+\delta_{2}-\varepsilon_{1}\right)-\lambda & 0 & a_{2} b m \\
-v_{2}+\delta_{2} & -\left(v_{2}+\varepsilon_{1}\right)-\lambda & -a_{2} b m \\
c a_{2} & 0 & -\lambda_{v}-\lambda
\end{array}\right|=0 \\
& =\left|\begin{array}{ccc}
-0.0034-\lambda & 0 & 0.0400 \\
-0.0014 & -0.0015-\lambda & -0.0400 \\
0.1920 & 0 & -(0.071+\lambda)
\end{array}\right|=0
\end{aligned}
$$

The corresponding characteristic equation is given by

$$
\lambda^{3}+a_{1} \lambda^{2}+a_{2} \lambda+a_{3}=0
$$

Where

$$
\begin{aligned}
& a_{1}=0.0759, a_{2}=-0.0073, a_{3}=-5.4634 \times 10^{-4} \\
& \Rightarrow \lambda^{3}+0.0759 \lambda^{2}-0.0073 \lambda-5.4634 \times 10^{-4}=0
\end{aligned}
$$

Now we apply the array of Routh-Hurwitz stability criteria. If all the sign of the first column have the same sign, thus all the roots have the negative real parts. This shows that the DFE point $(0,1,0)$ is stable. Now to check stability we draw the following array:

\begin{tabular}{|l|l|l|l|}
\hline$\lambda^{3}$ & \multicolumn{1}{|c|}{1} & -0.0073 & 0 \\
\hline$\lambda^{2}$ & 0.0759 & $-5.4634 \times 10^{-5}$ & 0 \\
\hline$\lambda^{1}$ & $b_{1}$ & $b_{2}$ & 0 \\
\hline$\lambda^{0}$ & $k_{1}$ & $k_{3}$ & 0 \\
\hline
\end{tabular}

And we can calculate out all the coefficients:

$$
\begin{aligned}
& b_{1}=\frac{-1}{0.0759}\left|\begin{array}{cc}
1 & -0.0073 \\
0.0759 & -5.4634 \times 10^{-5}
\end{array}\right|=-9.7101 \times 10^{-5} \\
& b_{2}=\frac{-1}{0.0759}\left|\begin{array}{cc}
1 & 0 \\
0.0759 & 0
\end{array}\right|=0 \\
& k_{1}=\frac{-1}{-9.7101 \times 10^{-5}}\left|\begin{array}{cc}
0.0759 & -5.4634 \times 10^{-4} \\
-9.7101 \times 10^{-5} & 0
\end{array}\right|=-5.4634 \times 10^{-4} \\
& k_{3}=\frac{-1}{-9.7101 \times 10^{-5}}\left|\begin{array}{cc}
0.07591 & 0 \\
-9.7101 \times 10^{-5} & 0
\end{array}\right|=0
\end{aligned}
$$

And filling these values into our Routh Array, we can determine whether the system is stable or not: 


\begin{tabular}{|l|l|l|l|}
\hline$\lambda^{3}$ & 1 & -0.0073 & 0 \\
\hline$\lambda^{2}$ & 0.00759 & $-5.4634 \times 10^{-4}$ & 0 \\
\hline$\lambda^{1}$ & $-9.7101 \times 10^{-4}$ & 0 & 0 \\
\hline$\lambda^{0}$ & $-5.4634 \times 10^{-4}$ & 0 & 0 \\
\hline
\end{tabular}

Since all the coefficients in the first column of the R-H table all have no the same sign and there is sign change, then the system is unstable.

Since the first columns do not contain the same sign, then by Routh-Hurwitz stability criteria the DFE point is unstable. Using Matlab we obtain the values of the Eigen value as follows.

The roots of the equation is

$$
\lambda_{1} 0.0852, \lambda_{2}=-0.0889, \lambda_{3}=-0.0722
$$

\subsection{Local Stability analysis of EE point of second age group}

The endemic equilibrium solution of (3.5.15)-(3.5.17) can be found by setting the right hand side to zero and solving the nonlinear system to obtain:

$$
\begin{aligned}
& a_{2}{ }^{b m} S_{h 2} i_{v}-\left(r_{2}+\delta_{2}-\varepsilon_{1}\right) i_{h 2}-\varepsilon_{1} i h_{1}+\delta_{2} i_{h 2}^{2}=0 \\
& -a_{2}{ }^{b m} S_{h 2} i_{v 2}-\varepsilon_{1} S_{h 1}+\delta_{2} S_{h 2} i_{h 2}-\varepsilon_{2} S_{h 2}+v_{2}\left(1-S_{h 2}-i_{h 2}\right)=0(\mathrm{~b}) \\
& c a_{2}\left(1-i_{v 2}\right) i_{h 2}-\lambda_{v} i_{v 2}=0 \text { (c) }
\end{aligned}
$$

Now from (c) we have that $i_{v 1}=\frac{a_{2} c i_{h 2}}{a_{2} c i_{h 2}+\lambda_{v}}$ (i)

Now substitute (i) in (a) and (b) solve for ih2*.

\section{$\Rightarrow \mathrm{ih} 2 *=0.02068$}

Finally substitute the value of ih $2 *=-0.2068$ in (a) we obtain

$(i h 2 * *, s h 2 * *, i v 2 * *)=(0.2068,-0.0513,0.3587)$. Then the Jacobian matrix of the model at any point $(i h 2 * *, \operatorname{sh} 2 * *, i v 2 * *)$ is given by

$J\left(\boldsymbol{i}_{h 2}^{*}, \boldsymbol{S}_{h 2} *, \boldsymbol{i}_{v 2}^{*}\right)=$

$$
\left[\begin{array}{ccc}
-\left(\boldsymbol{r}_{2}+\boldsymbol{\delta}_{2}-\boldsymbol{\varepsilon}_{1}\right)+2 \boldsymbol{\delta}_{2} \boldsymbol{S}_{h 2} & \boldsymbol{a}_{2} b m \boldsymbol{i}_{v 2} & a_{2}{ }^{b m} \boldsymbol{S}_{h 2} \\
-\boldsymbol{v}_{2}+\delta_{2} \boldsymbol{S}_{h 2} & -a_{2} b m \boldsymbol{i}_{v 2}+\boldsymbol{\delta}_{2} \boldsymbol{i}_{h 2}-\boldsymbol{\varepsilon}_{1}-\boldsymbol{v}_{2} & -a_{2}{ }^{b m} \boldsymbol{S}_{h 2} \\
c a_{2}\left(1-\boldsymbol{i}_{v 2}\right) & 0 & -c a_{2} \boldsymbol{i}_{h 2}-\lambda_{v}
\end{array}\right]
$$

Then the Jacobian matrix of the model at EE point $(i h 2 * *, s h 2 * *, i v 2 * *)=(0.2068,-0.0513,0.3587)$ is given by

$$
\begin{aligned}
& J\left(\boldsymbol{i}_{h 2}{ }^{*}, \boldsymbol{S}_{h 2}{ }^{*}, \boldsymbol{i}_{v 2}{ }^{*}\right)= \\
& {\left[\begin{array}{ccc}
-\left(r_{2}+\delta_{2}-\varepsilon_{1}\right)+2 \delta_{2}(-0.0513) & a_{2} b m(0.3587) & a_{2} b m(-0.0513) \\
-v_{2}+\delta_{2}(-0.0513) & -a_{2} b m(0.3587)+\delta_{2}(0.2068)-\varepsilon_{1}-v_{2} & -a_{2} b m(-0.0513) \\
c a_{2}(1-0.3587) & 0 & -c a_{2}(0.2068)-\lambda
\end{array}\right]} \\
& J(0.2068,-0.0513,0.3587)=\left[\begin{array}{ccc}
-0.0034 & 0.0143 & 0.0021 \\
-0.0014 & 0.0129 & -0.0021 \\
0.1231 & 0 & -0.1107
\end{array}\right]
\end{aligned}
$$

The characteristic polynomial is given by $\left|J-\lambda I_{3}\right|=0$ 


$$
\Rightarrow\left|\begin{array}{ccc}
-0.0034-\lambda & 0.143 & 0.0021 \\
-0.0014 & 0.0129-\lambda & -0.0021 \\
0.1231 & 0 & -(0.1107+\lambda)
\end{array}\right|=0
$$

The characteristic equation are given by $\lambda^{3}+0.1270 \lambda^{2}-0.0013 \lambda+5.5762 \times 10^{-6}$

Now we apply the array of Routh-Hurwitz stability criteria. If all the sign of the first column have the same sign, thus all the roots have the negative real parts. This shows that the EE point $(0.2068,-0.0513,0.3587)$ is stable. Now to check stability we draw the following array:

\begin{tabular}{|l|l|l|l|}
\hline$\lambda^{3}$ & \multicolumn{1}{|c|}{1} & -0.0013 & 0 \\
\hline$\lambda^{2}$ & 0.1270 & $5.5762 \times 10^{-6}$ & 0 \\
\hline$\lambda^{1}$ & $b_{1}$ & $b_{2}$ & 0 \\
\hline$\lambda^{0}$ & $k_{1}$ & $k_{2}$ & 0 \\
\hline
\end{tabular}

And we can calculate out all the coefficients:

$$
\begin{aligned}
& b_{1}=\frac{-1}{0.1270}\left|\begin{array}{cc}
1 & -0.0013 \\
0.1270 & -5.5762 \times 10^{-6}
\end{array}\right|=-0.0013 \\
& b_{2}=\frac{-1}{0.1270}\left|\begin{array}{cc}
1 & 0 \\
0.1270 & 0
\end{array}\right|=0 \\
& k_{1}=\frac{-1}{-0.0013}\left|\begin{array}{cc}
0.0759 & -5.5762 \times 10^{-6} \\
-0.0013 & 0
\end{array}\right|=-5.5762 \times 10^{-6} \\
& k_{2}=\frac{-1}{-0.0013}\left|\begin{array}{cc}
0.1270 & 0 \\
-0.0013 & 0
\end{array}\right|=0
\end{aligned}
$$

And filling these values into our Routh Array, we can determine whether the system is stable or not:

\begin{tabular}{|l|l|l|l|}
\hline$\lambda^{3}$ & \multicolumn{1}{|c|}{1} & -0.0013 & 0 \\
\hline$\lambda^{2}$ & 0.1270 & $-5.5762 \times 10^{-6}$ & 0 \\
\hline$\lambda^{1}$ & -0.0013 & 0 & 0 \\
\hline$\lambda^{0}$ & $-5.5762 \times 10^{-6}$ & 0 & 0 \\
\hline
\end{tabular}

Since all the coefficients in the first column of the R-H table all have no the same sign and there is sign change, then the system is unstable.

Since the first columns do not contain the same sign, then by Routh-Hurwitz stability criteria the DFE point is unstable. Using Matlab we obtain the values of the Eigen value as follows.

The roots of the equation is

$$
\lambda_{1}=-0.1368, \lambda_{2}=0.0049+0.0041 i, \lambda_{3}=0.0049-0.0041 i
$$

\subsection{Numerical simulation of the third age group model}

To determine the reproduction number and stability of age group three we consider the following model

$$
\begin{aligned}
& \frac{d i_{h 3}}{d t}=a_{3} b m S_{h 3} i_{v 2}-\left(r_{3}+\delta_{3}+\varepsilon_{2}\right) i_{h 3}+\varepsilon_{2} i_{h 2}+\delta_{3} i_{h 3}^{2} \\
& \frac{d S_{h 3}}{d t}=-a_{3} b m S_{h 3} i_{v 3}-\varepsilon_{2} S_{h 3}+\varepsilon_{2} S_{h 2}+\varepsilon_{2} S_{h 2}+v_{3}\left(1-S_{h 3}-i_{h 3}\right)+\delta_{3} S_{h 3} i_{h 3}
\end{aligned}
$$




$$
\frac{d i_{v 1}}{d t}=c a_{3}\left(1-i_{v 3}\right) i_{h 3}-\lambda_{v} i_{v 3}
$$

\subsection{The reproduction number of third age group}

From the given model of age group model (3.5.18)-(3.5.20) we obtain the reproduction number:

$$
\begin{aligned}
& R_{02}{ }^{2}=\frac{a_{3}{ }^{2} b c m}{m 11 \lambda v}, \text { or } R_{02}=\sqrt{\frac{a_{3}{ }^{2} b m c}{m 11 \lambda v}} \\
& \Rightarrow R_{02}=\sqrt{\frac{(0.2)^{2}(0.5)(0.48)(0.2)}{(0.071)(0.01+0.00000000667+0.0000502)}}=1.6463>1
\end{aligned}
$$

This indicate that the disease parasite in the community but less than the first age group.

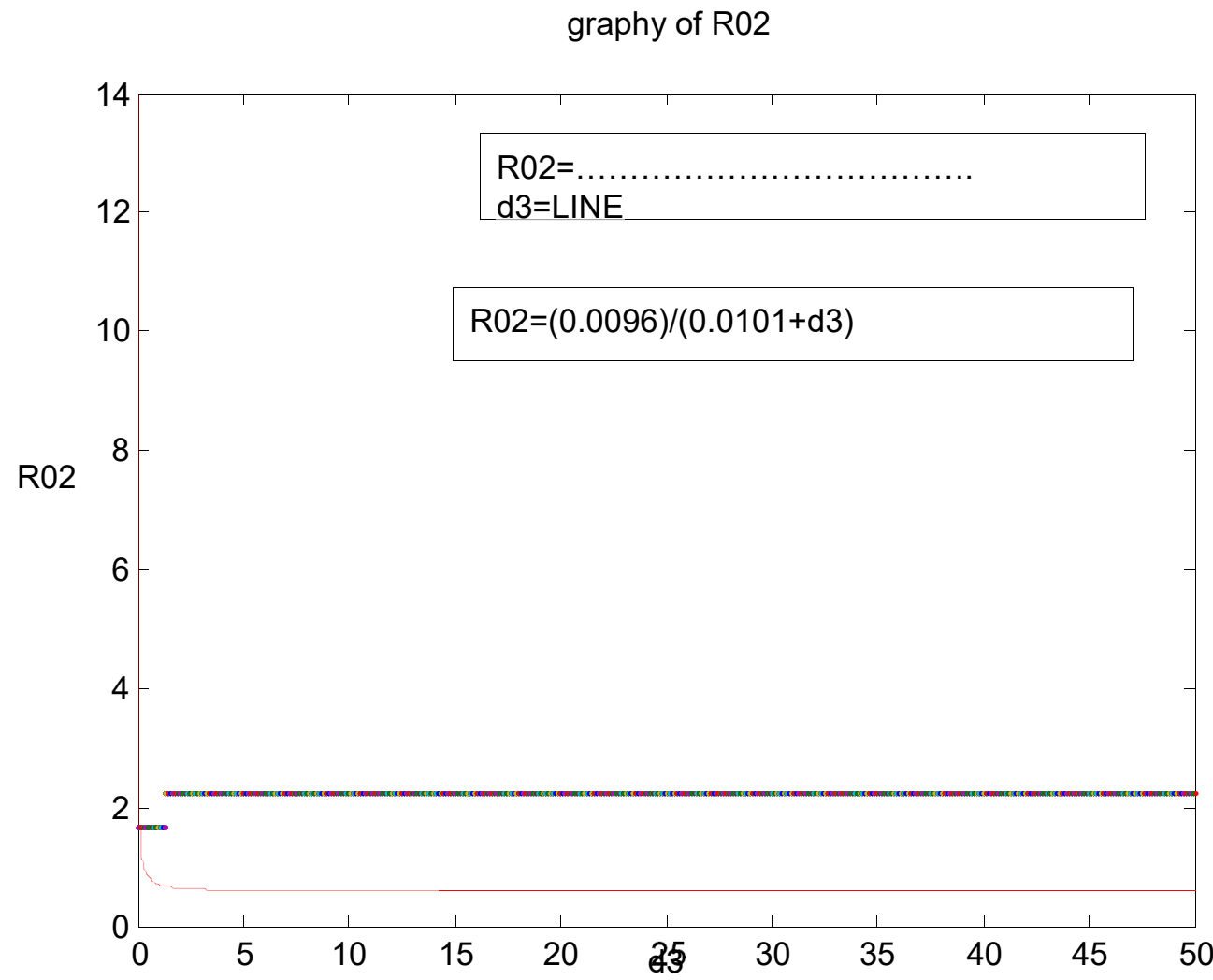

Figure 5.3 graph of the basic reproduction number $R_{02}$

From this graph we can observe that similar approach $R_{0}$ and phenomena observed. If the Per capita death rate $\delta_{3}$ increases then the reproduction number $R_{0}$ decreases. This means that when the Per capita death rate $\delta_{3}$ decrease then, the reproduction number is increasing and the susceptible population increasing. And when the Per capita death rate $\delta_{3}$ increases $R_{02}$ decreases that means when the when the Per capita death rate increase then the number of infected population decrease since individual in the infected class dies out.

4.10. Stability analysis of DFE point of third age group

The Jacobian matrix of the dynamical system (3.5.18)-(3.5.20) at any point (ih2*,sh2*,iv2*) is 


$$
\begin{aligned}
& J\left(i_{h 3}^{*}, s_{h 3}^{*}, i_{v}^{*}\right)= \\
& {\left[\begin{array}{ccc}
-\left(r_{3}+\delta_{3}+\varepsilon_{2}\right)+2 \delta_{3} i_{h 3} & a_{3} b m i_{v} & a_{3} b m s_{h 3} \\
-v_{3}+\delta_{3} s_{h 3} & -a_{3} b m i_{v}+\delta_{3} i_{h 3}-\varepsilon_{2}-v_{3} & -a_{3} b m s_{h 3} \\
c a_{3}\left(1-i_{v}\right) & 0 & -a_{3} c i_{h 3}-\lambda_{v}
\end{array}\right]}
\end{aligned}
$$

Then the Jacobian matrix of the above matrix at DFE point $(0,1,0)$ is given by

$$
J(0,1,0)=\left[\begin{array}{ccc}
-\left(r_{3}+\delta_{3}+\varepsilon_{2}\right) & 0 & a_{3} b m \\
-v_{3}+\delta_{3} & -\left(v_{3}+\varepsilon_{2}\right) & -a_{3} b m \\
a_{3} c & 0 & -\lambda_{v}
\end{array}\right] \text { (ii) }
$$

If we substitute the values of parameters in the given Jacobian matrix

$$
J(0,1,0)=\left[\begin{array}{ccc}
-0.0101 & 0 & 0.0200 \\
-7.9999 \times 10^{-4} & -8.5052 \times 10^{-4} & -0.0200 \\
0.0960 & 0 & -0.071
\end{array}\right]
$$

The characteristic polynomial is given by

$$
\left|\begin{array}{ccc}
-0.0101-\lambda & 0 & 0.0200 \\
-7.9999 \times 10^{-4} & -8.5020 \times 10^{-4}-\lambda & -0.0200 \\
0.0960 & 0 & -(0.071+\lambda)
\end{array}\right|=0 \text { (iii) }
$$

The corresponding characteristic polynomial is

$\lambda^{3}+d_{3} \lambda^{2}+d_{2} \lambda+d_{1}=0$ where

$d_{3}=6.0968 \times 10^{-7}-1.6154 \times 10^{-6}=-1.0257 \times 10^{-6}$

$d_{2}=7.1710 \times 10^{-4}+8 . .5870 \times 10^{-6}+6.0364 \times 10^{-5}=-0.0011$

$d_{1}=0.0101+0.071+8.5020 \times 10^{-4}=0.0819$

Now we apply the array of Routh-Hurwitz stability criteria. If all the sign of the first column have the same sign, thus all the roots have the negative real parts. This shows that the DFE point $(0,1,0)$ is stable. Now to check whether the DFE point $(0,1,0)$ is stability we draw the following array

\begin{tabular}{|l|l|l|l|}
\hline$\lambda^{3}$ & \multicolumn{1}{|c|}{1} & 0.0819 & 0 \\
\hline$\lambda^{2}$ & -0.0011 & $a_{4}=-1.0257 \times 10^{-6}$ & 0 \\
\hline$\lambda^{1}$ & $b_{1}$ & $b_{3}$ & 0 \\
\hline$\lambda^{0}$ & $k_{1}$ & $k_{3}$ & 0 \\
\hline
\end{tabular}

Where

$$
\begin{aligned}
& b_{1}=\frac{-1}{-0.0011}\left|\begin{array}{cc}
1 & 0.0819 \\
-0.0011 & -1.0257 \times 10^{-6}
\end{array}\right|=0.0810 \\
& b_{2}=\frac{-1}{-0.0011}\left|\begin{array}{cc}
1 & 0 \\
-0.0011 & 0
\end{array}\right|=0 \\
& k_{1}=\frac{-1}{0.0810}\left|\begin{array}{cc}
-0.0011 & -1.0257 \times 10^{-6} \\
0.0810 & 0
\end{array}\right|=-1.0257 \times 10^{-6} \\
& k_{2}=\frac{-1}{0.0810}\left|\begin{array}{cc}
-.0 .0661 & 0 \\
0.0097 & 0
\end{array}\right|=0
\end{aligned}
$$

And final we substitute 


\begin{tabular}{|l|l|l|l|}
\hline$\lambda^{3}$ & \multicolumn{1}{|c|}{1} & 0.0819 & 0 \\
\hline$\lambda^{2}$ & -0.0011 & $a_{4}=-1.0257 \times 10^{-6}$ & 0 \\
\hline$\lambda^{1}$ & 0.0810 & 0 & 0 \\
\hline$\lambda^{0}$ & $-1.0257 \times 10^{-6}$ & 0 & 0 \\
\hline
\end{tabular}

Where

From this array, we can clearly see that all of the signs of the first column do not contain positive value, there are sign changes,then by Routh-Hurwitz stability criteria the dynamical system of the given model is unstable.

Using Matlab we obtain

Roots :

$$
\lambda_{1}-0.0371, \lambda_{2}=0.0298, \lambda_{3}=-0.0009
$$

\subsection{Local Stability analysis of EE point of third age group}

The endemic equilibrium solution of (3.5.18)-(3.5.20) can be found by setting the right hand side to zero and solving the nonlinear system to obtain:

$$
\begin{aligned}
& \frac{d i_{h 3}}{d t}=a_{3} b m_{S_{h 3} i_{v 2}}-\left(r_{3}+\delta_{3}+\varepsilon_{2}\right) i_{h 3}+\varepsilon_{2} i_{h 2}+\delta_{3} i_{h 3}^{2}(\mathrm{i}) \\
& \frac{d S_{h 3}}{d t}=-a_{3} b m_{S_{h 3}} i_{v 3}-\varepsilon_{2} S_{h 3}+\varepsilon_{2} S_{h 2}+\varepsilon_{2} S_{h 2}+v_{3}\left(1-S_{h 3}-i_{h 3}\right)+\delta_{3} S_{h 3} i_{h 3} \\
& \frac{d i_{v 1}}{d t}=c a_{3}\left(1-i_{v 3}\right) i_{h 3}-\lambda_{v} i_{v 3}
\end{aligned}
$$

Now from (c) we have that $i_{v}=\frac{a_{3} c i_{h 3}}{a_{3} c i_{h 3}+\lambda_{v}}$

Now substitute (iv) in (a) and (b) solve for ih $3 * . \Rightarrow$ ih $3 *=-0.1274$

Finally substitute the value of ih $3^{*=}=0.1274$ in (a) we obtain

$\left(i_{h 3}{ }^{*}, s_{h 3}{ }^{*}, i_{v}{ }^{*}\right)=(-0.1274,0.2281,0.2853)$. Then the Jacobian matrix of the model at any point $\left(i_{h 3}^{*}, s_{h 3}^{*}, i_{v}^{*}\right)$ is given by

$J\left(i_{h 3}^{*}, s_{h 3}^{*}, i_{v}^{*}\right)=$

$$
\left[\begin{array}{ccc}
-\left(r_{3}+\delta_{3}+\varepsilon_{2}\right)+2 \delta_{3} i_{h 3} * & a_{3} b m i_{v}^{*} & a_{3} b m s_{h 3}^{*} \\
-v_{3}+\delta_{3} s_{h 3} * & -a_{3} b m i_{v}^{*}+\delta_{3} i_{h 3}^{*}-\varepsilon_{2}-v_{3} & -a_{3} b m s_{h 3}^{*} \\
c a_{3}\left(1-i_{v}^{*}\right) & 0 & -a_{3} c i_{h 3} *-\lambda_{v}
\end{array}\right]
$$

The Jacobian matrix of the third age group at any point $\left(i_{h 3}{ }^{*}, s_{h 3}{ }^{*}, i_{v}{ }^{*}\right)=(-0.1274,0.2281,-0.2853)$ is given as

$$
J(-0.1274,0.2281,-0.2281)=\left[\begin{array}{ccc}
-0.0101 & -0.0057 & 0.0046 \\
8.0000 \times 10^{-4} & -0.0049 & -0.0046 \\
0.1234 & 0 & -0.0832
\end{array}\right]
$$

The characteristic polynomial $(*)$ is given by

$$
\left[\begin{array}{ccc}
-0.0101-\lambda & -0.0057 & 0.0046 \\
8.0000 \times 10^{-4} & -0.0049-\lambda & -0.0046 \\
0.1234 & 0 & -0.0832-\lambda
\end{array}\right]
$$

The usual characteristic equation of $\left|J-\lambda I_{3}\right|=0$ is given by 


$$
\begin{aligned}
& \left|\begin{array}{ccc}
-0.0101-\lambda & 0 & 0.0200 \\
-7.9999 \times 10^{-4} & -8.5020 \times 10^{-4}-\lambda & -0.0200 \\
0.0960 & 0 & -(0.071+\lambda)
\end{array}\right|=0 \\
& =\lambda^{3}+0.0982 \lambda^{2}+7.2985 \times 10^{-4} \lambda+1.3362 \times 10^{-6}=0
\end{aligned}
$$

Now we apply the array of Routh-Hurwitz stability criteria. If all the sign of the first column have the same sign, thus all the roots have the negative real parts. This shows that the EE point $(-0.1274,0.2281,-0.2853)$ is stable. Now to check whether the EEP(-0.1274,0.2281,-0.2853) is stability we draw the following array

\begin{tabular}{|l|l|l|l|}
\hline$\lambda^{3}$ & \multicolumn{1}{|c|}{1} & $7.2985 \times 10^{-4}$ & 0 \\
\hline$\lambda^{2}$ & 0.0982 & $1.3362 \times 10^{-6}$ & 0 \\
\hline$\lambda^{1}$ & $b_{1}$ & $b_{2}$ & 0 \\
\hline$\lambda^{0}$ & $k_{1}$ & $k_{2}$ & 0 \\
\hline
\end{tabular}

And we can calculate out all the coefficients:

$$
\begin{aligned}
& b_{1}=\frac{-1}{0.0982}\left|\begin{array}{cc}
1 & 7.2985 \times 10^{-4} \\
0.0982 & 1.3362 \times 10^{-6}
\end{array}\right|=7.1624 \times 10^{-4} \\
& b_{2}=\frac{-1}{0.0982}\left|\begin{array}{cc}
1 & 0 \\
0.0982 & 0
\end{array}\right|=0 \\
& k_{1}=\frac{-1}{7.1624 \times 10^{-4}}\left|\begin{array}{cc}
0.0982 & 1.3362 \times 10^{-6} \\
7.1624 \times 10^{-4} & 0
\end{array}\right|=1.3362 \times 10^{-6} \\
& k_{2}=\frac{-1}{7.1624 \times 10^{-4}}\left|\begin{array}{cc}
0.0982 & 0 \\
7.1624 \times 10^{-4} & 0
\end{array}\right|=0
\end{aligned}
$$

And filling this values in the routh array we have

\begin{tabular}{|l|l|l|l|}
\hline$\lambda^{3}$ & \multicolumn{1}{|c|}{1} & $7.2985 \times 10^{-4}$ & 0 \\
\hline$\lambda^{2}$ & 0.0982 & $1.3362 \times 10^{-6}$ & 0 \\
\hline$\lambda^{1}$ & $7.1624 \times 10^{-4}$ & 0 & 0 \\
\hline$\lambda^{0}$ & $1.3362 \times 10^{-6}$ & 0 & 0 \\
\hline
\end{tabular}

Since all the coefficients in the first column of the R-H table all have the same sign and there is no sign change, then EEP $(-0.1274,0.2281,-0.2853)$ is stable by Routh-Hurwitz stability criteria .Using Matlab we obtain the values of the Eigen value as follows. The roots of the equation is

$$
\lambda_{1}=-0.0903, \lambda_{2}=-0.0049, \lambda_{3}=-0.0030
$$

\section{DISCUSSION OF RESULT}

In this paper a mathematical model of the prevalence and transmission of malaria was studied. The standard SIR differential equation model was used to predict the transmission and spread of malaria. By analyzing the model we found, the threshold parameter, Ro found which the basic reproduction number.From our analysis, we observe that the disease free equilibrium is stable when the threshold parameter is less than unity. i.e if $R_{0}<1$, then the epidemic will not spread and when $R_{0}>1$ the disease was parasite in the population and hence become endemic. The model has two equilibrium points namely the disease free equilibrium point and endemic equilibrium points. The existence and stability of disease free equilibrium point, endemic equilibrium points and sensitivity analysis of the model where preformed. We developed a model for the spread of malaria disease by human host vector population. The model divided three age classes children (age 0-5 years), youngest population (age 6-25), the 
remaining age classes and three epidemiological classes susceptible, infected and recovered. The model incorporates death rate due to malaria infection, death rate due to natural, new born of population, infection contact rate depending on the local population densities. Births are assumed to be continuous throughout the year.

The model is not considered the migration and emigration of the population in to compartment and from compartment.

\section{REFERENCES}

1. Analysis of age-structured malaria transmission model.

2. Aneke, S.J., 2002, Mathematical modelling of drug resistant malaria parasites and vector populations, Mathematical Methods in the Applied Sciences, 90, 385 -396.

3. Aron, J.L., May, R.M., 1982. The population dynamics of malaria. In: Anderson, R.M. (Ed.), Population Dynamics of Infectious Diseases: Theory and Applications, pp. 139-179. Chapman \& Hall, London

4. Aron, J.L., 1988, Mathematical modelling of immunity to malaria, Mathematical Biosciences, 90, 385 -396.

5. C.Akukwe, Malaria and Tuberculosis: Forgotten Diseases, (2004),http://www.theperspective.org/2004/May/forgottendiseases

6. C. Castillo-Chavez, Z. Feng, and W. Huang, On the computation of Ro and its role on global stability, in: Castillo-Chavez C., Blower S., van den Driessche P., Krirschner D. and Yakubu A.A.(Eds), Mathematica Approaches for Emerging and Reemerging Infectious Diseases: An Introduction. The IMA Volumes in Mathematics and its Applications. Springer Verlag, New York, 125(2002), 229-250.

7. CDC-(Centers for Disease Control h ttp://www.cdc.gov/malaria/about/index.html

8. Chiyaka C., Tchuenche J.M., Garira W., and Dube S. A mathematical analysis of the effects of control strategies on the transmission dynamics of malaria. Appl.Math.Comput. 195(2):641-662, 2008.

9. C. Mac Cormack, (1984) "Human ecology and behavior in malaria control in tropical Africa". Bull. WHO 62, Supplpp 81-87.

10. Construction of next-generation matrices O. Diekmann et al.(1990).

11. Coutinho, F.A.B., Burattini, M.N., Lopez, L.F. and Massad, E.2005, An approximate threshold condition for non-autonomous system: an application to a vector-borne infection, Mathematics and Computers in Simulation, 70, 149- 158 .

12. Diekmann, O.; Heesterbeek, J. A. P.; Metz, J. A. J. (1990). "On the definition and the computation of the basic reproduction ratio $\mathrm{R}_{0}$ in models for infectious diseases in heterogeneous populations". Journal 28 (4): 365 382.

13. Dietz, K., Molineaux, L., Thomas, A., 1974. A malaria model tested in the African savannah. Bull. World Health Organ. 50, 347-357.

14. Dietz, K., Molineaux, L. and Thomas, A., 1974, A malaria model tested in the African Savannah, Bulletin of the World Health Organization, 50, $347-357$.

15. Diekmann, O., Heesterbeek J. A. P. and Metz J. A. J., "On the definition and the computation of the basic reproduction ratio Ro in models for infectious diseases in heterogeneous populations," J. Math. Bio. 28 (1990): 365.

16. Ellis F. Mckenzie, Why model malaria?, Parasitology Today, 16 (2000), pp. 511-516.

17. F. B. Agusto, J. M. Tchuenche, Control strategies for the spread of malaria in humans with variable attractiveness, Math. Popul. Stud. 20 (2013), no. 2, 82-100.

18. Freeman, J., Laserson, K.F., Petralanda, I. and Spielman, A., 1999, Effect of chemotherapy on malaria transmission among Yonomami Amerindians: simulated consquences of placebo treatment, The American Journal of Tropical Medicine and Hygiene, 60(5), $774-780$.

19. G.A. Ngwa, W.S. Shu, A mathematical model for endemic malaria with variable human and mosquito populations, Mathematics and Computer Modelling 32 (2000) 747-763.

20. Gemperli, A., Vounatsou, P., Sogoba, N. and Smith, T., 2006, Malaria mapping using transmission models: application to survey data, American Journal of Epidemiology, 163, 289- 297.

21. Greenwood BM, Bojang K, Whitty CJM, Targett GA. Malaria. Lancet. 2005 Apr 23-29; 365 (9469):148798. 